\title{
HYPERKÄHLER MANIFOLDS OF JACOBIAN TYPE
}

\author{
MINGMIN SHEN
}

\begin{abstract}
In this paper we define the notion of a hyperkähler manifold (potentially) of Jacobian type. If we view hyperkähler manifolds as "abelian varieties", then those of Jacobian type should be viewed as "Jacobian varieties". Under a minor assumption on the polarization, we show that a very general polarized hyperkähler fourfold $F$ of $K 3^{[2]}$-type is not of Jacobian type. As a potential application, we conjecture that if a cubic fourfold is rational then its variety of lines is of Jacobian type. Under some technical assumption, it is proved that the variety of lines on a rational cubic fourfold is potentially of Jacobian type. We also prove the Hodge conjecture in degree 4 for a generic $F$ of $K 3^{[2]}$-type.
\end{abstract}

\section{INTRODUCTION}

The study of weight one Hodge structures leads to the theory of abelian varieties. Among all principally polarized abelian varieties, there is a special class called Jacobian varieties which corresponds to Hodge structures of curves. A corresponding theory for higher weight Hodge structures is still missing. In this paper, we propose a possible direction of a theory of "Jacobians" for weight two Hodge structures.

We do not know yet what the "principally polarized abelian varieties" for weight two Hodge structures should be. The most typical weight two Hodge structure is $\mathrm{H}^{2}(S, \mathbb{Z})$ where $S$ is a smooth projective surface, which is equipped with the intersection blinear form. A "p.p.a.v." in weight two should be a variety $X$ whose cohomology group $\mathrm{H}^{2}(X, \mathbb{Z})$ carries a natural nondegenerate bilinear form that is compatible with the Hodge structure. We know one class of such varieties, namely the hyperkähler manifolds $F$ whose cohomology group $\mathrm{H}^{2}(F, \mathbb{Z})$ carries the canonical Beauville-Bogomolov bilinear form, see [1, [10. Examples of such manifolds include generalized Kummer varieties, Hilbert scheme of points on a $K 3$ surface and the variety of lines on a smooth cubic fourfold.

To motivate the definition of "Jacobians" in weight two, we recall a characterization of Jacobian of curves. Let $(A, \Theta)$ be a principally polarized abelian variety of dimension $g$. Then a result of Matsusaka [17 says that $A$ is a Jacobian if and only if there exists a curve $f: C \rightarrow A$ such that $f_{*}[C]=\frac{[\Theta]^{g-1}}{(g-1) !}$ is the 1-dimensional minimal cohomology class. Or in other words, $A$ is a Jacobian if and only if the 1dimensional minimal cohomology class $\frac{[\Theta]^{g-1}}{(g-1) !}$ is effective. We define the "Jacobian" in weight two as follows.

Date: November 5, 2018.

2010 Mathematics Subject Classification. 14C30, 14E08, 14F25.

Key words and phrases. hyperkähler manifolds, Hodge classes, cubic fourfolds, rationality.

The author is supported by Simons Foundation as a Simons Postdoctoral Fellow. 
Definition 1.1. Let $F$ be a hyperkähler manifold of dimension $2 n$. Let $b(-,-)$ be the Beauville-Bogomolov bilinear form on $\mathrm{H}^{2}(F, \mathbb{Z})$. Let

$$
\operatorname{Alg}^{2 p}(F)=\operatorname{Im}\left(c l: \mathrm{CH}^{p}(F) \rightarrow \mathrm{H}^{2 p}(F, \mathbb{Z})\right), \quad \operatorname{Hdg}^{2 p}(F)=\mathrm{H}^{2 p}(F, \mathbb{Z}) \cap \mathrm{H}^{p, p}(F)
$$

be the subgroups of algebraic and Hodge classes. We define the transcendental lattice of $F$ to be

$$
\mathrm{H}^{2}(F, \mathbb{Z})_{\mathrm{tr}}=\operatorname{Alg}^{2}(F)^{\perp}
$$

with the restriction of the bilinear form $b$. A minimal class is an element $\theta \in$ $\mathrm{H}^{4 n-4}(F, \mathbb{Z})$ such that

$$
\left(\theta \cdot \alpha_{1} \cdot \alpha_{2}\right)_{F}=b\left(\alpha_{1}, \alpha_{2}\right), \quad \forall \alpha_{1}, \alpha_{2} \in \mathrm{H}^{2}(F, \mathbb{Z})_{\mathrm{tr}}
$$

A minimal Hodge class is a minimal class that is also a Hodge class. A projective hyperkähler manifold $F$ is of Jacobian type if there exists a surface $f: S \rightarrow F$ such that $f_{*}[S]$ is a minimal class.

By definition, a necessary condition for $F$ to be of Jacobian type is that it admits a minimal Hodge class. It is easy to see that if $F=S^{[n]}$ for some $K 3$-surface $S$, then $F$ is of Jacobian type. In fact, we can fix $n-1$ general points on $S$ and let the $n^{\text {th }}$ point vary and get a surface $\tilde{S} \subset F$. We can take $\theta$ to be the cycle class of $\tilde{S}$ and verify that $F$ is of Jacobian type. From now on, we will restrict ourselves to the case where $F$ is of $K 3^{[2]}$-type, i.e. deformation equivalent to $S^{[2]}$. The following definitions will be useful throughout this article.

Definition 1.2. Let $F$ be a hyperkähler manifold of $K 3^{[2]}$-type. Let $b(-,-)$ be the Beauville-Bogomolov bilinear form on $\mathrm{H}^{2}(F, \mathbb{Z})$. An element $\alpha \in \mathrm{H}^{2}(F, \mathbb{Z})$ is primitive if it is not divisible (in $\mathrm{H}^{2}(F, \mathbb{Z})$ ) by any integer greater than 1 . A primitive element $\alpha \in \mathrm{H}^{2}(F, \mathbb{Z})$ is even if $b\left(\alpha, \alpha^{\prime}\right) \in 2 \mathbb{Z}$, for all $\alpha^{\prime} \in \mathrm{H}^{2}(F, \mathbb{Z})$; otherwise, $\alpha$ is called odd. An element $\delta \in \mathrm{H}^{2}(F, \mathbb{Z})$ is exceptional if (i) $b(\delta, \delta)=-2$ and (ii) $\delta$ is even. Let $\Omega(F) \subset \mathrm{H}^{2}(F, \mathbb{Z})$ be the set of all exceptional elements. We use $\Omega_{0}(F)$ to denote $\Omega(F) /\{ \pm 1\}$. A polarization $\lambda_{0} \in \operatorname{Pic}(F)$ of $F$ is the class of an ample divisor. We say that $\left(F, \lambda_{0}\right)$ is primitively polarized if furthermore $\lambda_{0}$ is primitive.

Remark 1.3. An exceptional class $\delta$ is always primitive and satisfies $b\left(\delta, \mathrm{H}^{2}(F, \mathbb{Z})\right)=$ $2 \mathbb{Z}$. Let $\delta^{\perp}$ be the orthogonal complement (with respect to $b(-,-)$ ) of $\delta$. Then $\left(\delta^{\perp}, b\right)$ is isomorphic to the $K 3$-lattice and

$$
\mathrm{H}^{2}(F, \mathbb{Z})=\mathbb{Z} \delta \oplus \delta^{\perp} .
$$

We will show that if $\alpha$ is even, then $\alpha^{2}-\delta^{2}$ is divisible by 8 in $\mathrm{H}^{4}(F, \mathbb{Z})$, for all $\delta \in \Omega(F)$.

For a primitively polarized hyperkähler manifold, $\left(F, \lambda_{0}\right)$, of $K 3^{[2]}$-type, we will refer to the following technical assumption frequently.

Assumption 1.4. Either the polarization $\lambda_{0}$ is odd or it is even and $\frac{10+b\left(\lambda_{0}, \lambda_{0}\right)}{8}$ is an even integer.

The first main result of this paper is that a generic deformation of $S^{[2]}$ is not of Jacobian type; see Corollary 4.6.

Theorem 1.5. Let $\left(F, \lambda_{0}\right)$ be a primitively polarized hyperkähler manifold of $K 3^{[2]}$ type which satisfies Assumption 1.4. If $F$ has a minimal Hodge class, then $\operatorname{Pic}(F)$ 
has rank at least 2. In particular, a very general such $\left(F, \lambda_{0}\right)$ has no minimal Hodge class and hence is not of Jacobian type.

Our motivation for this study is the rationality problem of cubic fourfolds. Works concerning this problem include [8, [13] and [12]. In their famous paper [5], Clemens and Griffiths proved that a smooth cubic threefold is not rational by showing that its intermediate Jacobian is not a Jacobian. The key point here is that if a 3 dimensional algebraic variety is rational, then its intermediate Jacobian is the Jacobian of a curve. As an analogue, we make the following

Conjecture 1.6. Let $X \subset \mathbb{P}_{\mathrm{C}}^{5}$ be a smooth cubic fourfold and $F(X)$ its variety of lines. If $X$ is rational, then $F(X)$ is of Jacobian type.

We have two known classes of rational cubic fourfolds, namely the Pfaffian cubic fourfolds obtained by Beauville-Donagi [2] and the rational cubic fourfolds containing a plane obtained by Hassett [9]. In both cases, the above conjecture can be easily verified; see Remark 6.7. In [8], B. Hassett defines a cubic fourfold to be special if it contains a surface which is not homologous to a complete intersection.

Proposition 1.7. Let $X$ be a smooth cubic fourfold and $F=F(X)$ its variety of lines with $g_{1}$ being the Plücker polarization on $F$. Then the polarization $\lambda_{0}=g_{1}$ satisfies Assumption 1.4. If $F$ is of Jacobian type, then $X$ is special.

Then the following proposition follows immediately.

Proposition 1.8. If $X$ is a very general cubic fourfold, then $F(X)$ is not of Jacobian type. Conjecture 1.6 implies that a rational cubic fourfold is special.

To give further evidence of the Conjecture 1.6, we need the following definition.

Definition 1.9. Let $F$ be a hyperkähler manifold. We say that $F$ is potentially of Jacobian type if there exists a smooth surface $S$ (not necessarily irreducible) and a correspondence $\Gamma \in \mathrm{CH}_{2}(S \times F)$, such that

$$
\left([\Gamma]^{*} \alpha \cdot[\Gamma]^{*} \beta\right)_{S}=b(\alpha, \beta), \quad \forall \alpha, \beta \in \mathrm{H}^{2}(F, \mathbb{Z})_{\mathrm{tr}} .
$$

Theorem 1.10. Let $X$ be a smooth cubic fourfold and $F$ its variety of lines. If there is a birational map $f: \mathbb{P}^{4} \rightarrow X$ whose indeterminacy can be resolved by a simple successive blow-up, then $F$ is potentially of Jacobian type.

For the definition of simple successive blow-up, see Definition 6.4.

The plan of this paper is as follows. For a hyperkähler manifold $F$ of $K 3^{[2]}$-type, our investigation relies on an explicit basis for $\mathrm{H}^{4}(F, \mathbb{Z})$ obtained in Theorem 2.17 The following canonical torsion group

$$
\mathcal{T}^{4}(F)=\mathrm{H}^{4}(F, \mathbb{Z}) / \operatorname{Sym}^{2}(\Lambda), \quad \Lambda=\mathrm{H}^{2}(F, \mathbb{Z})
$$

comes into the picture in a subtle way. We give a description of this group in Theorem 3.4. In section 4, we first give an explicit description of a certain subgroup $V_{\lambda_{0}} \subset \operatorname{Hdg}^{4}(F)$ of integral Hodge classes; see Definition 4.1 for the notation. This is the technical Lemma 4.3 which becomes quite useful later on. We show that a minimal Hodge class in $\mathrm{H}^{4}(F, \mathbb{Z})$ is always contained in the linear span of intersections of divisors and the Beauville-Bogomolov form. In particular, if $F$ has Picard rank one then a minimal Hodge class is always in $V_{\lambda_{0}}$. From this combined with the technical lemma mentioned above, we deduce Theorem 1.5. In section 5 , we carry out an infinitesimal deformation calculation and show that on a very 
general $F$ the Hodge classes in degree 4 are generated by the polarization and the Beauville-Bogomolov form, i.e. $\operatorname{Hdg}^{4}(F)=V_{\lambda_{0}}$; see Theorem 5.1. This yet gives a second proof of the fact that a very general $F$ is not of Jacobian type. One consequence of this calculation is a proof of the Hodge conjecture for very general $F$. If $F$ is the variety of lines on a very general cubic fourfold, we are able to prove the integral Hodge conjecture thanks to the rich geometry that we have in this case; see Theorem 5.5. Section 7 gives a proof of Theorem 1.10. This section is independent of the previous ones.

Before ending this introduction, we would like to make a few remarks on some open questions. We see that $S^{[n]}$ is of Jacobian type, but we do not know whether the converse holds true or not. Namely, if we know that a hyperkähler manifold $F$ (deformation equivalent to $S^{[n]}$ ) is of Jacobian type, can we conclude that $F$ is birational to $S^{[n]}$, for some $K 3$ surface $S$ ? If $F=F(X)$ is the variety of lines on a cubic fourfold, then there is always the surface $S_{l}$ of lines meeting a given general line $l$ and the natural inclusion

$$
f: S_{l} \rightarrow F
$$

such that

$$
f^{*} \alpha \cdot f^{*} \beta=2 b(\alpha, \beta), \quad \forall \alpha, \beta \in \mathrm{H}^{2}(F, \mathbb{Z})_{\mathrm{tr}} .
$$

In analogy with the theory of abelian varieties, this is saying that $F(X)$ is a "Prym variety". In fact we do have a Prym construction as follows. There is an involution $\sigma$ on $S_{l}$ and the image of the restriction $\mathrm{H}^{2}(F, \mathbb{Z})_{\operatorname{tr}} \rightarrow \mathrm{H}^{2}\left(S_{l}, \mathbb{Z}\right)_{\operatorname{tr}}$ is the part on which $\sigma=-1$, see [11. If we replace $l$ by a rational curve of higher degree, then we get a Prym-Tjurin construction, see [21. We would like to ask the following questions. For a hyperkähler manifold $F$, what is the smallest integer $e>0$ such that there is a surface $f: S \rightarrow F$ satisfying

$$
f^{*} \alpha \cdot f^{*} \beta=e b(\alpha, \beta), \quad \forall \alpha, \beta \in \mathrm{H}^{2}(F, \mathbb{Z})_{\mathrm{tr}} ?
$$

If $S$ is a surface satisfying the above condition, is there a Prym-Tjurin construction on $S$ to give the restriction of $\mathrm{H}^{2}(F, \mathbb{Z})_{\mathrm{tr}}$ ?

Acknowledgement. The author would like to thank Claire Voisin for many helpful email correspondences. He also thanks D. Huybrechts, B. Totaro and E. Markman for their comments on an earlier version of this paper and C. Vial for many helpful discussions.

\section{The Degree 4 Integral Cohomology Group}

In this section, we fix $F$ to be a hyperkähler manifold which is deformation equivalent to the Hilbert scheme of length 2 subschemes of a $K 3$ surface. Such an $F$ will be called a hyperkähler manifold of $K 3^{[2]}$-type. We will give an explicit description of $\mathrm{H}^{4}(F, \mathbb{Z})$. Some general results about the integral cohomology ring were obtained in [19, [14] and [15].

Let $(\Lambda, b)$ be the lattice $\mathrm{H}^{2}(F, \mathbb{Z})$ equipped with the Beauville-Bogomolov bilinear form. We define the second symmetric power of $\Lambda$ to be

$$
\operatorname{Sym}^{2}(\Lambda)=\Lambda \otimes \Lambda /\langle a \otimes b-b \otimes a\rangle .
$$

We will simply use $\alpha \beta \in \operatorname{Sym}^{2}(\Lambda)$ to denote the image of $\alpha \otimes \beta \in \Lambda \otimes \Lambda$. The cup product

$$
\cup: \mathrm{H}^{2}(F, \mathbb{Z}) \otimes \mathrm{H}^{2}(F, \mathbb{Z}) \rightarrow \mathrm{H}^{4}(F, \mathbb{Z})
$$


naturally factors through $\operatorname{Sym}^{2}(\Lambda)$ and induces a homomorphism

$$
\rho: \operatorname{Sym}^{2}(\Lambda) \rightarrow \mathrm{H}^{4}(F, \mathbb{Z}), \quad \alpha \beta \mapsto \alpha \cup \beta .
$$

Lemma 2.1. (i) $\rho \otimes \mathbb{Q}: \operatorname{Sym}^{2}\left(\mathrm{H}^{2}(F, \mathbb{Q})\right) \rightarrow \mathrm{H}^{4}(F, \mathbb{Q})$ is an isomorphism.

(ii) $\rho$ is injective and the image has finite index in $\mathrm{H}^{4}(F, \mathbb{Z})$.

(iii) The intersection form on $\mathrm{H}^{4}(F, \mathbb{Z})$ restricted to $\operatorname{Sym}^{2} \Lambda$ is given by

$$
\rho\left(\alpha_{1} \alpha_{2}\right) \cdot \rho\left(\alpha_{3} \alpha_{4}\right)=b\left(\alpha_{1}, \alpha_{2}\right) b\left(\alpha_{3}, \alpha_{4}\right)+b\left(\alpha_{1}, \alpha_{3}\right) b\left(\alpha_{2}, \alpha_{4}\right)+b\left(\alpha_{1}, \alpha_{4}\right) b\left(\alpha_{2}, \alpha_{3}\right)
$$

for all $\alpha_{1}, \ldots, \alpha_{4} \in \Lambda$.

Proof. By a result of Verbitsky [23, 3], we know that the subalgebra of $\mathrm{H}^{*}(F, \mathbb{Q})$ generated by $\mathrm{H}^{2}(F, \mathbb{Q})$ is isomorphic to its symmetric algebra modulo an ideal supported at degrees of at least 6 . Hence $\operatorname{Sym}^{2} \mathrm{H}^{2}(F, \mathbb{Q}) \subset \mathrm{H}^{4}(F, \mathbb{Q})$. Namely $\rho$ is injective. At the same time, we know that $b_{4}(F)=276=\operatorname{dim} \operatorname{Sym}^{2} H^{2}(F)$; see 2. This implies that $\rho \otimes \mathbb{Q}$ is surjective and hence (i) follows. Statement (ii) is a direct consequence of (i) and (iii) is simply the Fujiki relation; see [1].

Definition 2.2. We define $\mathcal{T}^{4}(F)$ to be the quotient of $\mathrm{H}^{4}(F, \mathbb{Z})$ by $\rho\left(\operatorname{Sym}^{2} \Lambda\right)$.

Note that the group $\mathcal{T}^{4}(F)$ is always a torsion group of finite order.

2.1. The special case of $F=S^{[2]}$. In this subsection, we carry out some explicit computations in the situation $F=S^{[2]}$ for some $K 3$ surface $S$. Let $\tau: Z \rightarrow S \times S$ be the blow up of $S \times S$ along the diagonal $\Delta_{S}: S \rightarrow S \times S$. Let $j: \tilde{\Delta} \hookrightarrow Z$ be the exceptional divisor of the blow-up. There is a natural morphism $\eta: \tilde{\Delta} \rightarrow S$ that realizes $\tilde{\Delta}$ as a $\mathbb{P}^{1}$-bundle over $S$. Actually, $\tilde{\Delta}=\mathbb{P}\left(T_{S}\right)$ is the projectivization of the tangent bundle of $S$. There is a natural degree 2 finite morphism $\pi: Z \rightarrow S^{[2]}$ that ramifies along the divisor $\tilde{\Delta}$. Let $G=\{1, \sigma\} \cong \mathbb{Z} / 2 \mathbb{Z}$ act on $S \times S$ by switching the two factors. Then this action lifts to an action of $G$ on $Z$ and $S^{[2]}$ is the associated quotient. Let $\Delta=\pi(\tilde{\Delta}) \subset S^{[2]}$. By construction, $\left.\pi\right|_{\tilde{\Delta}}: \tilde{\Delta} \rightarrow \Delta$ is an isomorphism. We use $j^{\prime}$ to denote the inclusion of $\Delta$ into $S^{[2]}$. In summary, we have the following picture,

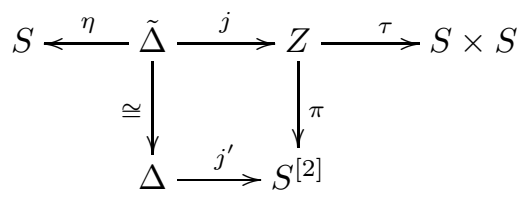

The blow up $\tau$ gives a short exact sequence

$$
0 \longrightarrow \mathrm{H}^{2}(S \times S, \mathbb{Z}) \stackrel{\tau^{*}}{\longrightarrow} \mathrm{H}^{2}(Z, \mathbb{Z}) \stackrel{\cdot(-f)}{\longrightarrow} \mathbb{Z} \longrightarrow 0 .
$$

The element $f \in \mathrm{H}^{6}(Z, \mathbb{Z})$ is the class of a fiber of $\eta: \tilde{\Delta} \rightarrow S$. This sequence naturally splits since we have a splitting homomorphism $\mathbb{Z} \rightarrow \mathrm{H}^{2}(Z, \mathbb{Z}), 1 \mapsto[\tilde{\Delta}]$. Hence we have a canonical isomorphism

$$
\mathrm{H}^{2}(Z, \mathbb{Z}) \cong \mathrm{H}^{2}(S \times S, \mathbb{Z}) \oplus \mathbb{Z}[\tilde{\Delta}] .
$$

This is compatible with the $G$-action ( $G$ acts trivially on $[\tilde{\Delta}])$. Note that by Künneth formula we have

$$
\mathrm{H}^{2}(S \times S, \mathbb{Z})=p_{1}^{*} \mathrm{H}^{2}(S, \mathbb{Z}) \oplus p_{2}^{*} \mathrm{H}^{2}(S, \mathbb{Z}) \cong \mathrm{H}^{2}(S, \mathbb{Z}) \otimes \mathbb{Z}[G] .
$$


From now on, we will fix a $\mathbb{Z}$-basis

$$
\left\{\mathfrak{a}_{1}, \mathfrak{a}_{2}, \ldots, \mathfrak{a}_{22}\right\}
$$

for $\mathrm{H}^{2}(S, \mathbb{Z})$. The following lemma follows from the above computations

Lemma 2.3. (i) $\mathrm{H}^{2}(Z, \mathbb{Z})^{G}=\oplus_{i=1}^{22} \mathbb{Z} \tau^{*}\left(p_{1}^{*} \mathfrak{a}_{i}+p_{2}^{*} \mathfrak{a}_{i}\right) \oplus \mathbb{Z}[\tilde{\Delta}]$.

(ii) For all odd $i$, we have $\mathrm{H}^{i}\left(G, \mathrm{H}^{2}(Z, \mathbb{Z})\right)=0$.

(iii) For all even positive $i$, we have $\mathrm{H}^{i}\left(G, \mathrm{H}^{2}(Z, \mathbb{Z})\right)=\mathbb{Z} / 2 \mathbb{Z}$.

We have similar descriptions of $\mathrm{H}^{4}(Z, \mathbb{Z})$. First we have the following exact sequence (derived from the Leray spectral sequence associated to $\tau: Z \rightarrow S \times S$ ),

$$
0 \longrightarrow \mathrm{H}^{4}(S \times S, \mathbb{Z}) \longrightarrow \mathrm{H}^{4}(Z, \mathbb{Z}) \stackrel{\phi}{\longrightarrow} \mathrm{H}^{2}\left(S, R^{2} \eta_{*} \mathbb{Z}\right) \longrightarrow 0 .
$$

Let $\xi=c_{1}\left(\mathcal{O}_{\tilde{\Delta}}(1)\right) \in \mathrm{H}^{2}(\tilde{\Delta}, \mathbb{Z})$, where $\mathcal{O}_{\tilde{\Delta}}(1)$ is the relative $\mathcal{O}(1)$ bundle of $\tilde{\Delta}=$ $\mathbb{P}\left(T_{S}\right) \rightarrow S$. Then $\xi$ defines an isomorphism $\mathbb{Z} \cong R^{2} \eta_{*} \mathbb{Z}$. This induces

$$
\mathrm{H}^{2}(S, \mathbb{Z}) \cong \mathrm{H}^{2}\left(S, R^{2} \eta_{*} \mathbb{Z}\right) .
$$

Let $G$ act trivially on $\mathrm{H}^{2}\left(S, R^{2} \eta_{*} \mathbb{Z}\right)$, then the sequence (1) respects the $G$-actions.

Lemma 2.4. (i) The homomorphism $\mathrm{H}^{2}(S, \mathbb{Z}) \rightarrow \mathrm{H}^{4}(Z, \mathbb{Z}), \mathfrak{a}_{i} \mapsto-j_{*} \eta^{*} \mathfrak{a}_{i}$, splits the sequence (11) canonically.

(ii) As G-modules, we have

$$
\mathrm{H}^{4}(Z, \mathbb{Z})=\mathrm{H}^{4}(S \times S, \mathbb{Z}) \oplus \mathrm{H}^{2}(S, \mathbb{Z})
$$

where $G$ acts trivially on the factor $\mathrm{H}^{2}(S, \mathbb{Z})$.

Proof. The homomorphism $\phi: \mathrm{H}^{4}(Z, \mathbb{Z}) \rightarrow \mathrm{H}^{2}\left(S, R^{2} \eta_{*} \mathbb{Z}\right)$ factors as

$$
\mathrm{H}^{4}(Z, \mathbb{Z}) \stackrel{j^{*}}{\longrightarrow} \mathrm{H}^{4}(\tilde{\Delta}, \mathbb{Z}) \stackrel{\eta_{*}}{\longrightarrow} \mathrm{H}^{2}(S, \mathbb{Z}) .
$$

It is easy to check that

$$
\begin{aligned}
\eta_{*} j^{*}\left(-j_{*} \eta^{*} \mathfrak{a}\right) & =-\eta_{*}\left(j^{*}[\tilde{\Delta}] \cdot \eta^{*} \mathfrak{a}\right) \\
& =-\eta_{*}\left(-\xi \cdot \eta^{*} \mathfrak{a}\right) \\
& =\eta_{*}\left(\xi \cdot \eta^{*} \mathfrak{a}\right) \\
& =\mathfrak{a}, \quad \forall \mathfrak{a} \in \mathrm{H}^{2}(S, \mathbb{Z}),
\end{aligned}
$$

where $\xi \in \mathrm{H}^{2}(\tilde{\Delta}, \mathbb{Z})$ is the first Chern class of the relative $\mathcal{O}(1)$ bundle. This proves (i). The statement (ii) follows directly from (i).

Lemma 2.5. (i) The group $\mathrm{H}^{0}\left(G, \mathrm{H}^{4}(S \times S, \mathbb{Z})\right)$ is freely generated by $p_{1}^{*}[p t]+p_{2}^{*}[p t]$, $\left\{p_{1}^{*} \mathfrak{a}_{i} \otimes p_{2}^{*} \mathfrak{a}_{i}\right\}_{i=1}^{22}$ and $\left\{p_{1}^{*} \mathfrak{a}_{i} \otimes p_{2}^{*} \mathfrak{a}_{j}+p_{1}^{*} \mathfrak{a}_{j} \otimes p_{2}^{*} \mathfrak{a}_{i}\right\}_{1 \leq i<j \leq 22}$.

(ii) For all odd $i$, we have $\mathrm{H}^{i}\left(G, \mathrm{H}^{4}(S \times S, \mathbb{Z})\right)=0$; for all even $i>0$, we have $\mathrm{H}^{i}\left(G, \mathrm{H}^{4}(S \times S, \mathbb{Z})\right) \cong(\mathbb{Z} / 2 \mathbb{Z})^{22}$.

(iii) The group $\mathrm{H}^{0}\left(G, \mathrm{H}^{4}(Z, \mathbb{Z})\right)$ is freely generated by $e_{0}=\tau^{*}\left(p_{1}^{*}[p t]+p_{2}^{*}[p t]\right)$, $\left\{e_{i}=\tau^{*}\left(p_{1}^{*} \mathfrak{a}_{i} \otimes p_{2}^{*} \mathfrak{a}_{i}\right)\right\}_{i=1}^{22}, \quad\left\{e_{i j}=\tau^{*}\left(p_{1}^{*} \mathfrak{a}_{i} \otimes p_{2}^{*} \mathfrak{a}_{j}+p_{1}^{*} \mathfrak{a}_{j} \otimes p_{2}^{*} \mathfrak{a}_{i}\right)\right\}_{1 \leq i<j \leq 22}$ and $\left\{u_{i}^{\prime}=j_{*} \eta^{*} \mathfrak{a}_{i}\right\}_{1 \leq i \leq 22}$.

(iv) For all odd $i$, we have $\mathrm{H}^{i}\left(G, \mathrm{H}^{4}(Z, \mathbb{Z})\right)=0$; for all even $i>0$, we have $\mathrm{H}^{i}\left(G, \mathrm{H}^{4}(Z, \mathbb{Z})\right) \cong(\mathbb{Z} / 2 \mathbb{Z})^{44}$. 
Proof. Statement (i) follows from the Künneth formula $\mathrm{H}^{4}(S \times S, \mathbb{Z})=\mathrm{H}^{4}(S, \mathbb{Z}) \otimes \mathrm{H}^{0}(S, \mathbb{Z}) \oplus \mathrm{H}^{2}(S, \mathbb{Z}) \otimes \mathrm{H}^{2}(S, \mathbb{Z}) \oplus \mathrm{H}^{0}(S, \mathbb{Z}) \otimes \mathrm{H}^{4}(S, \mathbb{Z})$.

Then (iii) follows easily from (i) and Lemma 2.4. By direct calculation of the group cohomology groups, we get (ii) and (iv).

We will use the following spectral sequences frequently.

Proposition 2.6 (Grothendieck, chapter V of [7]). Let $X$ be a topological space with an action by a finite group $G$. Let $Y=X / G$ be the quotient and $\pi: X \rightarrow Y$ the natural map. Let $\Gamma^{G}(-)$ be the covariant functor from the category of $G$-sheaves on $X$ to the category of abelian groups which sends a $G$-sheaf $\mathscr{F}$ to the $G$-invariant sections $\mathrm{H}^{0}(X, \mathscr{F})^{G}$. The right derived functor of $\Gamma^{G}$ is denoted $\mathrm{H}^{i}(G ; X, \mathbb{Z})$. Then there are two spectral sequences associated to the situation,

$$
{ }_{I} E_{2}^{p, q}=\mathrm{H}^{p}\left(Y, R^{q}\left(\pi_{*}^{G}\right) \mathscr{F}\right) \Longrightarrow \mathrm{H}^{p+q}(G ; X, \mathscr{F})
$$

and

$$
{ }_{I I} E_{2}^{p, q}=\mathrm{H}^{p}\left(G, \mathrm{H}^{q}(X, \mathscr{F})\right) \Longrightarrow \mathrm{H}^{p+q}(G ; X, \mathscr{F})
$$

where $\pi_{*}^{G}(\mathscr{F})=\left(\pi_{*} \mathscr{F}\right)^{G}$ for all $G$-sheaves $\mathscr{F}$.

Now we apply the second spectral sequence to the special case $\pi: Z \rightarrow F=Z / G$ and $\mathscr{F}=\mathbb{Z}$. Note that $\mathrm{H}^{i}(Z, \mathbb{Z})=0$ for odd $i$. This together with Lemma 2.5 force the spectral sequence to degenerate at the ${ }_{I I} E_{2}$ page. Thus we get the following lemma.

Lemma 2.7. (i) There is a short exact sequence

$$
0 \longrightarrow \mathbb{Z} / 2 \mathbb{Z} \longrightarrow \mathrm{H}^{2}(G ; Z, \mathbb{Z}) \longrightarrow \mathrm{H}^{2}(Z, \mathbb{Z})^{G} \longrightarrow 0
$$

(ii) We also have the following short exact sequence

$$
0 \longrightarrow T_{1} \longrightarrow \mathrm{H}^{4}(G ; Z, \mathbb{Z}) \longrightarrow \mathrm{H}^{4}(Z, \mathbb{Z})^{G} \longrightarrow 0,
$$

where $T_{1}$ is a torsion group of order 4 .

Consider the first spectral sequence ${ }_{I} E$. Direct calculation of the stalks gives

$$
R^{i} \pi_{*}^{G} \mathbb{Z}= \begin{cases}\mathbb{Z}, & i=0 \\ 0, & i \text { odd; } \\ (\mathbb{Z} / 2 \mathbb{Z})_{\Delta}, & i>0 \text { even. }\end{cases}
$$

This creates enough zeros in the ${ }_{I} E_{2}$ page and forces the spectral sequence to degenerate at this page. Hence we have the following lemma.

Lemma 2.8. (i) There is a short exact sequence

$$
0 \longrightarrow \mathrm{H}^{2}\left(S^{[2]}, \mathbb{Z}\right) \longrightarrow \mathrm{H}^{2}(G ; Z, \mathbb{Z}) \longrightarrow \mathrm{H}^{0}(\Delta, \mathbb{Z} / 2 \mathbb{Z}) \longrightarrow 0 .
$$

(ii) We also have a short exact sequence

$$
0 \longrightarrow \mathrm{H}^{4}\left(S^{[2]}, \mathbb{Z}\right) \longrightarrow \mathrm{H}^{4}(G ; Z, \mathbb{Z}) \longrightarrow T_{2} \longrightarrow 0,
$$

where $T_{2}$ is torsion group that fits into the following exact sequence

$$
0 \longrightarrow \mathrm{H}^{2}(\Delta, \mathbb{Z} / 2 \mathbb{Z}) \longrightarrow T_{2} \longrightarrow \mathrm{H}^{0}(\Delta, \mathbb{Z} / 2 \mathbb{Z}) \longrightarrow 0
$$

In particular, $T_{2}$ is of order $2^{24}$. 
Corollary 2.9 (Beauville, [1]). (i) The homomorphism $\pi^{*}: \mathrm{H}^{2}\left(S^{[2]}, \mathbb{Z}\right) \rightarrow \mathrm{H}^{2}(Z, \mathbb{Z})^{G}$ is an isomorphism and hence we have a canonical isomorphism

$$
\mathrm{H}^{2}\left(S^{[2]}, \mathbb{Z}\right)=\mathrm{H}^{2}(S, \mathbb{Z}) \oplus \mathbb{Z} \delta
$$

where $\delta$ satisfies $2 \delta=[\Delta]$.

(ii) There is a short exact sequence

$$
0 \longrightarrow \mathrm{H}^{4}\left(S^{[2]}, \mathbb{Z}\right) \stackrel{\pi^{*}}{\longrightarrow} \mathrm{H}^{4}(Z, \mathbb{Z})^{G} \longrightarrow T_{3} \longrightarrow 0
$$

where $T_{3} \cong(\mathbb{Z} / 2 \mathbb{Z})^{22}$.

Proof. To prove (i), we first note that the composition

$$
\mathrm{H}^{2}\left(G, \mathrm{H}^{0}(Z, \mathbb{Z})\right)=\mathbb{Z} / 2 \mathbb{Z} \rightarrow \mathrm{H}^{2}(G ; Z, \mathbb{Z}) \rightarrow \mathrm{H}^{0}\left(\Delta, R^{2} \pi_{*}^{G} \mathbb{Z}\right)=\mathbb{Z} / 2 \mathbb{Z}
$$

is an isomorphism. By easy diagram chasing, this forces the composition

$$
\mathrm{H}^{2}\left(S^{[2]}, \mathbb{Z}\right) \rightarrow \mathrm{H}^{2}(G ; Z, \mathbb{Z}) \rightarrow \mathrm{H}^{2}(Z, \mathbb{Z})^{G}
$$

to be an isomorphism. Hence there is an element $\delta \in \mathrm{H}^{2}\left(S^{[2]}, \mathbb{Z}\right)$ such that $\pi^{*} \delta=$ $[\tilde{\Delta}]$. Since $\pi^{*}[\Delta]=2[\tilde{\Delta}]$, we get $2 \delta=[\Delta]$. To prove (ii), we consider the following diagram

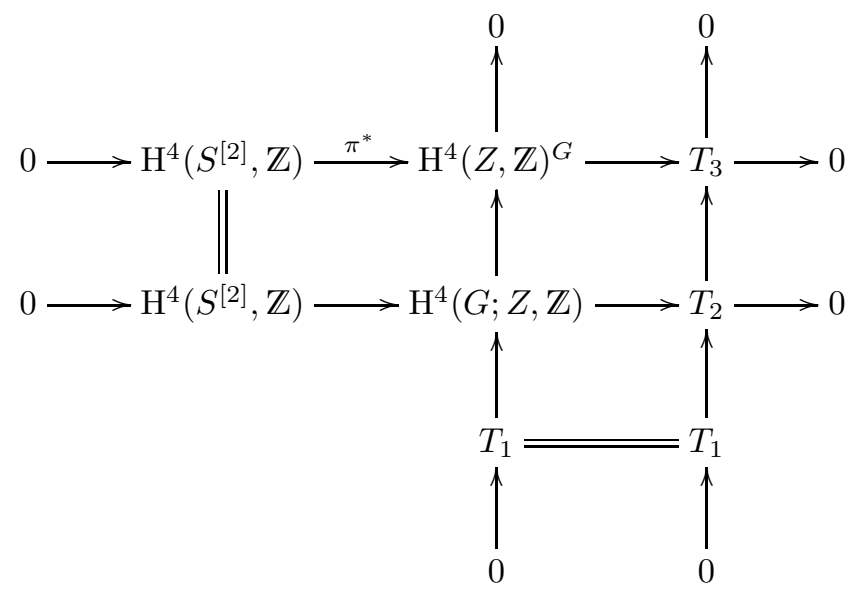

This implies that $T_{3}$ is of order $2^{22}$. For any $\alpha \in \mathrm{H}^{4}(Z, \mathbb{Z})^{G}$, we have $2 \alpha=\pi^{*}\left(\pi_{*} \alpha\right)$. This implies that $T_{3}$ is of 2 -torsion. Hence we have $T_{3} \cong(\mathbb{Z} / 2 \mathbb{Z})^{22}$.

Remark 2.10. For any $\mathfrak{a} \in \mathrm{H}^{2}(S, \mathbb{Z})$, its image in $\mathrm{H}^{2}(F, \mathbb{Z})$ will be denoted $\hat{\mathfrak{a}}$. Hence $\mathrm{H}^{2}\left(S^{[2]}, \mathbb{Z}\right)$ has a basis

$$
\left\{\hat{\mathfrak{a}}_{1}, \hat{\mathfrak{a}}_{2}, \ldots, \hat{\mathfrak{a}}_{22}, \delta\right\}
$$

The element $\hat{\mathfrak{a}}_{i}$ can be described as the unique element satisfying

$$
\pi^{*} \hat{\mathfrak{a}}_{i}=\tau^{*}\left(p_{1}^{*} \mathfrak{a}_{i}+p_{2}^{*} \mathfrak{a}_{i}\right) .
$$


Consider the following diagram

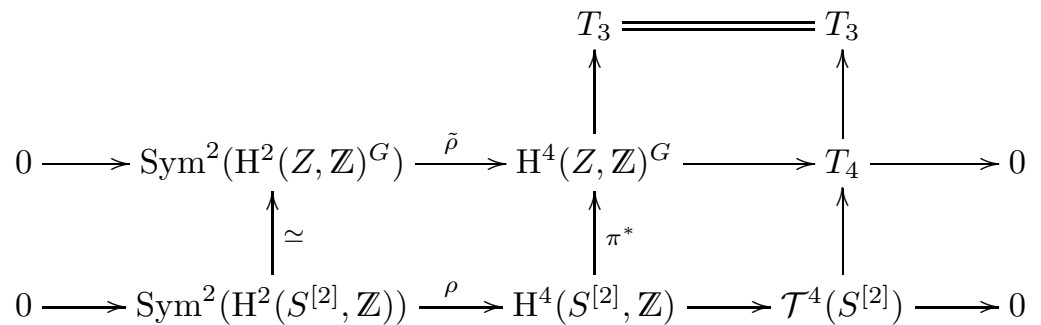

where all the 3 -terms columns are short exact. Use the canonical isomorphism in (i) of Lemma 2.3, we know that the image of $\tilde{\rho}$ is freely generated by

$$
\begin{aligned}
\tau^{*}\left(p_{1}^{*} \mathfrak{a}_{i}+p_{2}^{*} \mathfrak{a}_{i}\right) \cup \tau^{*}\left(p_{1}^{*} \mathfrak{a}_{i}+p_{2}^{*} \mathfrak{a}_{i}\right)= & \tau^{*}\left(p_{1}^{*}\left(\mathfrak{a}_{i} \cup \mathfrak{a}_{i}\right)+p_{2}^{*}\left(\mathfrak{a}_{i} \cup \mathfrak{a}_{i}\right)+2 p_{1}^{*} \mathfrak{a}_{i} \cup p_{2}^{*} \mathfrak{a}_{i}\right) \\
= & \left(\mathfrak{a}_{i} \cdot \mathfrak{a}_{i}\right) e_{0}+2 e_{i}, \quad 1 \leq i \leq 22 ; \\
\tau^{*}\left(p_{1}^{*} \mathfrak{a}_{i}+p_{2}^{*} \mathfrak{a}_{i}\right) \cup \tau^{*}\left(p_{1}^{*} \mathfrak{a}_{j}+p_{2}^{*} \mathfrak{a}_{j}\right)= & \tau^{*}\left(p_{1}^{*}\left(\mathfrak{a}_{i} \cup \mathfrak{a}_{j}\right)+p_{2}^{*}\left(\mathfrak{a}_{i} \cup \mathfrak{a}_{j}\right)+\right. \\
& \left.p_{1}^{*} \mathfrak{a}_{i} \cup p_{2}^{*} \mathfrak{a}_{j}+p_{1}^{*} \mathfrak{a}_{j} \cup p_{2}^{*} \mathfrak{a}_{i}\right) \\
= & \left(\mathfrak{a}_{i} \cdot \mathfrak{a}_{j}\right) e_{0}+e_{i j}, \quad 1 \leq i<j \leq 22 ; \\
\tau^{*}\left(p_{1}^{*} \mathfrak{a}_{i}+p_{2}^{*} \mathfrak{a}_{i}\right) \cup[\tilde{\Delta}]= & j_{*}\left(j^{*} \tau^{*}\left(p_{1}^{*} \mathfrak{a}_{i}+p_{2}^{*} \mathfrak{a}_{i}\right)\right) \\
= & j_{*}\left(2 \eta^{*} \mathfrak{a}_{i}\right) \\
= & 2 u_{i}^{\prime}
\end{aligned}
$$

and

$$
[\tilde{\Delta}]^{2}=j_{*} j^{*}[\tilde{\Delta}]=-j_{*} \xi
$$

where $\xi$ is the first Chern class of the relative $\mathcal{O}(1)$ bundle of $\tilde{\Delta}=\mathbb{P}\left(T_{S}\right) \rightarrow S$. The next lemma gives us

$$
j_{*} \xi=j_{*}\left(c_{1}(\mathcal{E})\right)=j_{*}\left(c_{1}(\mathcal{E}) \cap \eta^{*}[S]\right)=\tau^{*}\left[\Delta_{S}\right],
$$

where $\Delta_{S} \subset S \times S$ is the diagonal and $\mathcal{E}$ fits into the following short exact sequence

$$
0 \longrightarrow \mathcal{O}_{\tilde{\Delta}}(-1) \longrightarrow \eta^{*} T_{S} \longrightarrow \mathcal{E} \longrightarrow 0 \text {. }
$$

Lemma 2.11 ([6], Proposition 6.7). Let $X$ be a smooth projective variety and $Y \subset X$ a smooth subvariety of codimension d. Let $\tau: \tilde{X} \rightarrow X$ be the blow up of $X$ along $Y$ and $\tilde{Y}=\mathbb{P}\left(\mathscr{N}_{Y / X}\right) \subset \tilde{X}$ the exceptional divisor. Let $j: \tilde{Y} \rightarrow \tilde{X}$ be the inclusion, $\eta: \tilde{Y} \rightarrow Y$ the natural projection and $i: Y \rightarrow X$ the inclusion. On $\tilde{Y}$, we have the following short exact sequence

$$
0 \longrightarrow \mathcal{O}_{\tilde{Y}}(-1) \longrightarrow \eta^{*} \mathscr{N}_{Y / X} \longrightarrow \mathcal{E} \longrightarrow 0 .
$$

Then we have

$$
\tau^{*} i_{*}(x)=j_{*}\left(c_{d-1}(\mathcal{E}) \cap \eta^{*} x\right)
$$

for all $x$ in the cohomology or Chow groups of $Y$.

Let $A=\left(a_{i j}\right)_{22 \times 22}$, with $a_{i j}=\mathfrak{a}_{i} \cdot \mathfrak{a}_{j} \in \mathbb{Z}$, be the intersection matrix of $\mathrm{H}^{2}(S, \mathbb{Z})$. Since the intersection form is unimodular, we know that $B=A^{-1}$ is integral. 
Actually, let $\left\{\mathfrak{a}_{1}^{\vee}, \mathfrak{a}_{2}^{\vee}, \ldots, \mathfrak{a}_{22}^{\vee}\right\}$ be the dual basis of $\mathrm{H}^{2}(S, \mathbb{Z})^{\vee}$. The intersection form gives a canonical isomorphism $\mathrm{H}^{2}(S, \mathbb{Z})^{\vee} \cong \mathrm{H}^{2}(S, \mathbb{Z})$, under which we have

$$
\mathfrak{a}_{i}^{\vee}=\sum_{j=1}^{22} b_{i j} \mathfrak{a}_{j} .
$$

As a correspondence, $\Delta_{S}$ acts trivially on the cohomology of $S$; it follows that

$$
\left[\Delta_{S}\right]=\sum_{i=1}^{22} \mathfrak{a}_{i} \otimes \mathfrak{a}_{i}^{\vee}+[p t] \otimes[S]+[S] \otimes[p t] .
$$

This implies

$$
\tau^{*}\left[\Delta_{S}\right]=\sum_{1 \leq i<j \leq 22} b_{i j} e_{i j}+\sum_{i=1}^{22} b_{i i} e_{i}+e_{0} .
$$

This combined with (3) and (4) implies that

$$
[\tilde{\Delta}]^{2}=-\sum_{1 \leq i<j \leq 22} b_{i j} e_{i j}-\sum_{i=1}^{22} b_{i i} e_{i}-e_{0} .
$$

With the chosen basis, the matrix representation for $\tilde{\rho}$ is

$$
M_{\tilde{\rho}}=\left(\begin{array}{cccccccccc}
2 & & & & & & \vdots & & \\
& \ddots & & & & & a_{i i} & & \\
& & 2 & & & & \vdots & & \\
& & & 1 & & & \vdots & & \\
& & & & \ddots & & a_{i j} & & \\
& & & & & 1 & \vdots & & \\
\cdots & -b_{i i} & \cdots & \cdots & -b_{i j} & \cdots & -1 & & \\
& & & & & & & 2 & & \\
& & & & & & & & \ddots & \\
& & & & & & & & 2
\end{array}\right)
$$

In the matrix $M_{\tilde{\rho}}$, we first have 22 of "2"s on diagonal indexed by $(i, i), 1 \leq i \leq 22$; then we have 231 of "1"s indexed by $(i, j), 1 \leq i \leq j \leq 22$; after that there is a single " -1 " followed by 22 of " 2 " s. In the row (resp. column) corresponding to the diagonal entry "-1", we have all the entries $b_{i j}$ of $B$ sitting before (resp. $a_{i j}$ of $A$ sitting above) "-1". All the remaining entries of $M_{\tilde{\rho}}$ are "0"s. One checks that $\operatorname{det}\left(M_{\tilde{\rho}}\right)=5 \cdot 2^{45}$. Hence we have the following lemma.

Lemma 2.12. (i) The group $T_{4}$ is of order $5 \cdot 2^{45}$.

(ii) The group $\mathcal{T}^{4}\left(S^{[2]}\right)$ is of order $5 \cdot 2^{23}$.

Statement (ii) was first obtained in [4. Proposition 6.6]. Their method uses the isomorphism between $\mathrm{H}^{2}\left(S^{[2]}, \mathbb{Z}\right)$ and the lattice $U^{\oplus 3} \oplus E_{8}(-1)^{\oplus 2} \oplus\langle-2\rangle$ and a discriminant computation.

In [19, §4], the operators $L^{\lambda}$ were introduced to study the cohomology of Hilbert scheme of $n$ points on a surface, where $\lambda$ is a partition of $n$. In our case, we will 
use the operator $L^{1,1}$ which can be described explicitly as

$$
L^{1,1}(\mathfrak{a})=\frac{1}{2} \pi_{*}\left(\tau^{*}\left(p_{1}^{*} \mathfrak{a} \cup p_{2}^{*} \mathfrak{a}\right)-j_{*} \eta^{*} \mathfrak{a}\right) \in \mathrm{H}^{4}(F, \mathbb{Z}),
$$

for all $\mathfrak{a} \in \mathrm{H}^{2}(S, \mathbb{Z})$. The key point here is that $L^{1,1}$ is integral. When $\mathfrak{a}$ is the class of a curve $C \subset S$, then $L^{1,1}(\mathfrak{a})$ is represented by the closure of $\{\pi(x, y): x, y \in$ $C, x \neq y\}$. This implies that $L^{1,1}$ is integral on Hodge classes; see 19, Theorem 4.5]. The above equation (5) allows us to check the integrality of $L^{1,1}$ on a basis of $\mathrm{H}^{2}(S, \mathbb{Z})$. Thus we only need to check that for finitely many general elements $\mathfrak{a}$ of $\mathrm{H}^{2}(S, \mathbb{Z})$. For each of those $\mathfrak{a}$, by deforming the complex structure on $S$, we can arrange $\mathfrak{a}$ to be a Hodge class and hence we see that $L^{1,1}$ is integral on this $\mathfrak{a}$. It follows that $L^{1,1}$ is integral on all of those finitely many a's. Hence as an operator, $L^{1,1}$ is integral.

The following theorem will be very useful in explicit calculations.

Theorem 2.13. Let $\left\{\mathfrak{a}_{i}\right\}$ be an integral basis of $\mathrm{H}^{2}(S, \mathbb{Z})$ and $A$ the intersection matrix. Let $B=A^{-1}$. There is a basis

$$
\left\{v_{0},\left.v_{i}\right|_{1 \leq i \leq 22},\left.v_{i j}\right|_{1 \leq i<j \leq 22},\left.u_{i}\right|_{1 \leq i \leq 22}\right\}
$$

of $\mathrm{H}^{4}\left(S^{[2]}, \mathbb{Z}\right)$, such that

$$
\pi^{*} v_{0}=e_{0}, \quad \pi^{*} v_{i}=e_{i}-u_{i}^{\prime}, \quad \pi^{*} v_{i j}=e_{i j}, \quad \pi^{*} u_{i}=2 u_{i}^{\prime} .
$$

The cup product $\mathrm{H}^{2}\left(S^{[2]}, \mathbb{Z}\right) \times \mathrm{H}^{2}\left(S^{[2]}, \mathbb{Z}\right) \rightarrow \mathrm{H}^{4}\left(S^{[2]}, \mathbb{Z}\right)$ can be described explicitly as

$$
\begin{aligned}
\hat{\mathfrak{a}}_{i} \cdot \hat{\mathfrak{a}}_{j} & =v_{i j}+a_{i j} v_{0}, \quad 1 \leq i<j \leq 22 ; \\
\hat{\mathfrak{a}}_{i} \cdot \hat{\mathfrak{a}}_{i} & =2 v_{i}+u_{i}+a_{i i} v_{0}, \quad 1 \leq i \leq 22 ; \\
\hat{\mathfrak{a}}_{i} \cdot \delta & =u_{i}, \quad 1 \leq i \leq 22 ; \\
\delta \cdot \delta & =-\sum_{1 \leq i<j \leq 22} b_{i j} v_{i j}-\sum_{1 \leq i \leq 22} b_{i i} v_{i}-\sum_{1 \leq i \leq 22} \frac{b_{i i}}{2} u_{i}-v_{0} .
\end{aligned}
$$

In particular, $\hat{\mathfrak{a}}_{i}\left(\hat{\mathfrak{a}}_{i}-\delta\right)$ is divisible by 2 in $\mathrm{H}^{4}\left(S^{[2]}, \mathbb{Z}\right)$.

Proof. We take $u_{i}=j_{*}^{\prime} \eta^{*} \mathfrak{a}_{i}=\pi_{*} u_{i}^{\prime}, v_{0}=\pi_{*}\left(\tau^{*} p_{1}^{*}[p t]\right)$ and $v_{i j}=\pi_{*} \tau^{*}\left(p_{1}^{*} \mathfrak{a}_{i} \cup p_{2}^{*} \mathfrak{a}_{j}\right)$. We also set $v_{i}=L^{1,1}\left(\mathfrak{a}_{i}\right) \in \mathrm{H}^{4}\left(S^{[2]}, \mathbb{Z}\right)$. We also have the following relation

$$
e_{i}=\tau^{*}\left(p_{1}^{*} \mathfrak{a}_{i} \cup p_{2}^{*} \mathfrak{a}_{i}\right)=\pi^{*} L^{1,1}\left(\mathfrak{a}_{i}\right)+u_{i}^{\prime}, \quad 1 \leq i \leq 22 .
$$

Let $N \subset \mathrm{H}^{4}\left(S^{[2]}, \mathbb{Z}\right)$ be the subgroup generated by $v_{0},\left\{v_{i}\right\}_{i=1}^{22},\left\{v_{i j}\right\}_{1 \leq i<j \leq 22}$, $\left\{u_{i}\right\}_{i=1}^{22}$. By writing down explicitly the matrix of $\pi^{*}: N \rightarrow \mathrm{H}^{4}(Z, \mathbb{Z})^{G}$, we see that the cokernel of the above map has size $2^{22}$. By (ii) of Lemma 2.9, this implies that $N=\mathrm{H}^{4}\left(S^{[2]}, \mathbb{Z}\right)$. To check the formula of cup products, we only need to check the identities after pulling back via $\pi^{*}$. For example,

$$
\begin{aligned}
\pi^{*}\left(\hat{\mathfrak{a}}_{i} \cdot \hat{\mathfrak{a}}_{j}\right) & =\tau^{*}\left(p_{1}^{*} \mathfrak{a}_{i}+p_{2}^{*} \mathfrak{a}_{i}\right) \cdot \tau^{*}\left(p_{1}^{*} \mathfrak{a}_{j}+p_{2}^{*} \mathfrak{a}_{j}\right) \\
& =\tau^{*}\left(\left(\mathfrak{a}_{i} \cdot \mathfrak{a}_{j}\right) p_{1}^{*}[p t]+\left(\mathfrak{a}_{j} \cdot \mathfrak{a}_{i}\right) p_{2}^{*}[p t]\right)+\tau^{*}\left(p_{1}^{*} \mathfrak{a}_{i} \cup p_{2}^{*} \mathfrak{a}_{j}+p_{1}^{*} \mathfrak{a}_{j} \cup p_{2}^{*} \mathfrak{a}_{i}\right) \\
& =\pi^{*}\left(a_{i j} v_{0}+v_{i j}\right), \quad 1 \leq i<j \leq 22 .
\end{aligned}
$$

The other equalities are checked similarly. 
2.2. The general case. In this subsection, we assume that $F$ is a hyperkähler manifold of $K 3^{[2]}$-type. For any $\delta \in \Omega(F)$, then $\delta$ (or $-\delta$ ) essentially arises from some $S^{[2]}$ in the deformation equivalent family, see Lemma 3.4 of [16]. Hence for cohomological computations, it is harmless to automatically view an element $\delta \in \Omega(F)$ as coming from an isomorphism $F \cong S^{[2]}$.

We first prove the following

Lemma 2.14. Let $\delta, \delta^{\prime} \in \Omega(F)$, then the following are true.

(i) The class $\delta-\delta^{\prime}$ is divisible by 2 in $\mathrm{H}^{2}(F, \mathbb{Z})$.

(ii) The class $\delta^{2}-\delta^{\prime 2}$ is divisible by 8 in $\mathrm{H}^{4}(F, \mathbb{Z})$.

(iii) For any $\alpha \in \mathrm{H}^{2}(F, \mathbb{Z})$, the class $\alpha \cdot(\alpha-\delta) \in \mathrm{H}^{4}(F, \mathbb{Z})$ is divisible by 2 .

Proof. We assume that $F=S^{[2]}$ for some $K 3$-surface $S$. Let $\delta$ be the half of the boundary divisor. We use the notations in Theorem 2.13. Then we can write $\delta^{\prime}=\hat{\mathfrak{a}}^{\prime}-c \delta$, where $b\left(\hat{\mathfrak{a}}^{\prime}, \delta\right)=0$. Since $\delta^{\prime}$ is exceptional, we have $b\left(\delta^{\prime}, \alpha\right) \in 2 \mathbb{Z}$ for all $\alpha \in \mathrm{H}^{2}(F, \mathbb{Z})$. This forces $\hat{\mathfrak{a}}^{\prime}=2 \hat{\mathfrak{a}}$, for some $\hat{\mathfrak{a}}=\sum a_{i} \hat{\mathfrak{a}}_{i} \in \delta^{\perp}$. Since $\delta^{\prime}$ is primitive, we know that $c \in \mathbb{Z}$ is odd. This proves (i). For (ii), we write explicitly

$$
\begin{aligned}
\delta^{2}-\delta^{2} & =4 \hat{\mathfrak{a}}^{2}-4 c \delta \hat{\mathfrak{a}}+c^{2} \delta^{2} \\
& =4 \sum_{i=1}^{22}\left(a_{i}^{2} \hat{\mathfrak{a}}_{i}^{2}-c a_{i} \delta \hat{\mathfrak{a}}_{i}\right)+8 \sum_{1 \leq i<j \leq 22} a_{i} a_{j} \hat{\mathfrak{a}}_{i} \hat{\mathfrak{a}}_{j}+\left(c^{2}-1\right) \delta^{2} .
\end{aligned}
$$

Since $c^{2}-1$ is divisible by 8 , we only need to show that the first sum is divisible by 8 . This can be seen from

$$
4 \sum_{i=1}^{22}\left(a_{i}^{2} \hat{\mathfrak{a}}_{i}^{2}-c a_{i} \delta \hat{\mathfrak{a}}_{i}\right)=4 \sum a_{i}\left(\hat{\mathfrak{a}}_{i}^{2}-\delta \hat{\mathfrak{a}}_{i}\right)+8 \sum\left(\begin{array}{c}
a_{i} \\
2
\end{array}\right) \hat{\mathfrak{a}}_{i}^{2}-4(c-1) \sum a_{i} \delta \hat{\mathfrak{a}}_{i}
$$

and the fact that $c$ is odd and that $\hat{\mathfrak{a}}_{i}^{2}-\delta \hat{\mathfrak{a}}_{i}$ is divisible by 2 , see Theorem 2.13 Given (i), we only need to prove (iii) for the exceptional element $\delta \in \Omega$ coming from an isomorphism $F \cong S^{[2]}$. This case follows from Theorem 2.13

Definition 2.15. Let $\delta \in \Omega(F)$. We define a quadratic map $v_{\delta}: \mathrm{H}^{2}(F, \mathbb{Z}) \rightarrow$ $\mathrm{H}^{4}(F, \mathbb{Z})$ by putting

$$
v_{\delta}(\alpha)=\frac{1}{2} \alpha \cdot(\alpha-\delta)
$$

It is known that $\delta^{\perp}$ is the lattice of a $K 3$ surface. Let $\left\{\hat{\mathfrak{a}}_{1}, \ldots, \hat{\mathfrak{a}}_{22}\right\}$ be a basis for $\delta^{\perp}$ and let $A=\left(b\left(\hat{\mathfrak{a}}_{i}, \hat{\mathfrak{a}}_{j}\right)\right)_{22 \times 22}$ be the intersection matrix. Set $B=A^{-1}$. We define

$$
v_{0}(\delta)=\frac{1}{10}\left(\delta \cdot \delta+\frac{1}{2} \sum_{i, j=1}^{22} b_{i j} \hat{\mathfrak{a}}_{i} \cdot \hat{\mathfrak{a}}_{j}\right) .
$$

Remark 2.16. Let $F=S^{[2]}$ and notations be as in Theorem 2.13, then $v_{0}(\delta)$ is simply the class $v_{0}$. This can be deduced from a direct computation using the cup product formulas obtained in Theorem 2.13 In particular, $v_{0}(\delta) \in \mathrm{H}^{4}(F, \mathbb{Z})$ is integral.

Theorem 2.17. Let $\delta \in \Omega(F)$ and $\left\{\hat{\mathfrak{a}}_{1}, \ldots, \hat{\mathfrak{a}}_{22}\right\}$ be a basis of $\delta^{\perp}$. Then the cohomology group $\mathrm{H}^{4}(F, \mathbb{Z})$ admits an integral basis

$$
\left\{v_{0}(\delta),\left.\quad \hat{\mathfrak{a}}_{i} \cdot \hat{\mathfrak{a}}_{j}\right|_{1 \leq i \leq j \leq 22},\left.\quad v_{\delta}\left(\hat{\mathfrak{a}}_{i}\right)\right|_{1 \leq i \leq 22},\left.\quad \delta \cdot \hat{\mathfrak{a}}_{i}\right|_{1 \leq i \leq 22}\right\} .
$$




\section{A DESCRIPTION OF $\mathcal{T}^{4}(F)$}

Let $F$ be a hyperkähler manifold of $K 3^{[2]}$-type. This section is devoted to a canonical description of the group $\mathcal{T}^{4}(F)$. By Lemma 2.12(ii), we know that the order of the group $\mathcal{T}^{4}(F)$ is $5 \cdot 2^{23}$.

Lemma 3.1. Let $F$ be a hyperkähler manifold of $K 3^{[2]}$-type and $\delta \in \Omega(F)$ an exceptional class. Then

(i) The composition $\mathrm{H}^{2}(F, \mathbb{Z}) \stackrel{v_{\delta}}{\longrightarrow} \mathrm{H}^{4}(F, \mathbb{Z}) \longrightarrow \mathcal{T}^{4}(F)$ induces a homomorphism

$$
\bar{v}_{\delta}: \mathrm{H}^{2}(F, \mathbb{Z}) \otimes \mathbb{Z} / 2 \mathbb{Z} \rightarrow \mathcal{T}^{4}(F) .
$$

(ii) The homomorphism $\bar{v}_{\delta}$ is independent of the choice of $\delta$.

(iii) The image $\bar{\delta}$ of $\delta$ in $\mathrm{H}^{2}(F, \mathbb{Z}) \otimes \mathbb{Z} / 2 \mathbb{Z}$ is independent of the choice of $\delta$.

(iv) The kernel of $\bar{v}_{\delta}$ is generated by $\bar{\delta}$.

Remark 3.2. Since $\bar{v}_{\delta}$ is independent of the choice of $\delta$, we will simply write

$$
\bar{v}: \mathrm{H}^{2}(F, \mathbb{Z} / 2 \mathbb{Z}) \rightarrow \mathcal{T}^{4}(F)
$$

for this canonical homomorphism.

Proof. For (i), we note that

$$
\begin{aligned}
v_{\delta}\left(\hat{\mathfrak{a}}+\hat{\mathfrak{a}}^{\prime}\right) & =\frac{1}{2}\left(\hat{\mathfrak{a}}+\hat{\mathfrak{a}}^{\prime}\right)\left(\hat{\mathfrak{a}}+\hat{\mathfrak{a}}^{\prime}-\delta\right) \\
& =v_{\delta}(\hat{\mathfrak{a}})+v_{\delta}\left(\hat{\mathfrak{a}}^{\prime}\right)+\hat{\mathfrak{a}} \hat{\mathfrak{a}}^{\prime}
\end{aligned}
$$

which implies that the map $\mathrm{H}^{2}(F, \mathbb{Z}) \rightarrow \mathcal{T}^{4}(F)$ is a homomorphism. One easily checks that this homomorphism vanishes on $2 \mathrm{H}^{2}(F, \mathbb{Z})$ and hence (i) follows. If we pick another exceptional class $\delta^{\prime} \in \Omega(F)$, then

$$
v_{\delta^{\prime}}(\hat{\mathfrak{a}})-v_{\delta}(\hat{\mathfrak{a}})=\frac{1}{2}\left(\delta-\delta^{\prime}\right) \hat{\mathfrak{a}} .
$$

By Lemma 2.14 the element $\frac{\delta-\delta^{\prime}}{2}$ is integral. Thus $v_{\delta^{\prime}}(\hat{\mathfrak{a}})$ and $v_{\delta}(\hat{\mathfrak{a}})$ map to the same image in $\mathcal{T}^{4}(F)$, which proves (ii). Statement (iii) follows from the fact that $\delta-\delta^{\prime}$ is divisible by 2; see Lemma 2.14. By the definition of $\bar{v}_{\delta}$, we see that $\bar{\delta}$ is in the kernel. Let $\left\{\hat{\mathfrak{a}}_{1}, \ldots, \hat{\mathfrak{a}}_{22}\right\}$ be a basis of $\delta^{\perp}$. Then Theorem 2.13 implies that $\bar{v}_{\delta}\left(\hat{\mathfrak{a}}_{i}\right) \neq 0$ for all $i$. Since the basis is arbitrary, we get that $\bar{v}_{\delta}(\hat{\mathfrak{a}}) \neq 0$ for all primitive $\hat{\mathfrak{a}} \in \delta^{\perp}$. This implies that the images $\bar{v}_{\delta}\left(\hat{\mathfrak{a}}_{i}\right)$ are $\mathbb{Z} / 2 \mathbb{Z}$-linearly independent. This proves (iv).

Lemma 3.3. Let $F$ be a hyperkähler manifold of $K 3^{[2]}$-type and $\delta \in \Omega(F)$ an exceptional class. Then the following are true.

(i) $v_{0}(\delta) \in \mathrm{H}^{4}(F, \mathbb{Z})$ is the unique element satisfying the following

$$
\left(v_{0}(\delta) \cdot \alpha \cdot \beta\right)_{F}=b(\alpha, \beta)+\frac{1}{4} b(\delta, \alpha) b(\delta, \beta), \quad \forall \alpha, \beta \in \mathrm{H}^{2}(F, \mathbb{Z}) .
$$

(ii) The image $\bar{v}_{0}(\delta)$ of $v_{0}(\delta)$ in $\mathcal{T}^{4}(F)$ is an element of order 10 . For a different choice $\delta^{\prime} \in \Omega(F)$, the difference $\bar{v}_{0}(\delta)-\bar{v}_{0}\left(\delta^{\prime}\right)$ is an element in the image of $\bar{v}_{\delta}$. In particular, the element $\bar{w}_{0}=2 \bar{v}_{0}(\delta)$ is independent of the choice of $\delta$.

Proof. We take a basis $\left\{\hat{\mathfrak{a}}_{1}, \ldots, \hat{\mathfrak{a}}_{22}\right\}$ of $\delta^{\perp}$. To prove (i), we may work in the special case $F=S^{[2]}$. Then the $v_{0}$ in Theorem 2.13 is represented by a smooth surface 
$\tilde{S} \subset F$, which parameterizes all length two subschemes of $S$ containing a given point of $S$. Note that $\tilde{S}$ is the blow up of $S$ at that point. Then we have

$$
\left.\hat{\mathfrak{a}}_{i}\right|_{\tilde{S}}=\sigma^{*} \mathfrak{a}_{i},\left.\quad \delta\right|_{\tilde{S}}=E
$$

where $\sigma: \tilde{S} \rightarrow S$ is the blow up with $E$ being the exceptional curve. Since the class of $\tilde{S}$ on $F$ is simply $v_{0}$, we have

$$
\left(v_{0} \cdot \hat{\mathfrak{a}}_{i} \cdot \delta\right)_{F}=\left(\sigma^{*} \mathfrak{a}_{i} \cdot E\right)_{\tilde{S}}=0, \quad\left(v_{0} \cdot \delta^{2}\right)_{F}=E^{2}=-1,
$$

and

$$
\left(v_{0} \cdot \hat{\mathfrak{a}}_{i} \cdot \hat{\mathfrak{a}}_{j}\right)_{F}=\left(\sigma^{*} \mathfrak{a}_{i} \cdot \sigma^{*} \mathfrak{a}_{j}\right)_{\tilde{S}}=\left(\mathfrak{a}_{i} \cdot \mathfrak{a}_{j}\right)=b\left(\hat{\mathfrak{a}}_{i}, \hat{\mathfrak{a}}_{j}\right) .
$$

One easily checks that the equality in (i) holds for $\alpha, \beta$ from the basis $\left\{\hat{\mathfrak{a}}_{1}, \ldots, \hat{\mathfrak{a}}_{22}, \delta\right\}$. By linearity, (i) holds for all $\alpha, \beta$. For (ii), we may still assume $F=S^{[2]}$. First we note that by the definition of $v_{0}(\delta)$, we know that its image in $\mathcal{T}^{4}(F)$ is an element of order 10; see equation (7). In $\mathrm{H}^{4}(F, \mathbb{Z})$, we have the element

$$
q=\sum_{1 \leq i, j \leq 22} b_{i j} \hat{\mathfrak{a}}_{\mathfrak{i}} \hat{\mathfrak{a}}_{j}-\frac{1}{2} \delta^{2}
$$

where $B=\left(b_{i j}\right)$ is the inverse of the intersection matrix of $\left\{\mathfrak{a}_{1}, \ldots, \mathfrak{a}_{22}\right\}$. We know that $q$ is independent of the choice of the exceptional class $\delta$ and the basis $\left\{\hat{\mathfrak{a}}_{i}\right\}$ of $\delta^{\perp}$, see the discussion of next section. Then equation (7) can be written as

$$
v_{0}(\delta)=\frac{1}{8} \delta^{2}+\frac{1}{20} q .
$$

This implies that

$$
v_{0}\left(\delta^{\prime}\right)-v_{0}(\delta)=\frac{1}{8}\left(\delta^{\prime 2}-\delta^{2}\right) .
$$

As in the proof of Lemma 2.14, we can write $\delta^{\prime}=2 \hat{\mathfrak{a}}+c \delta$ for some $\hat{\mathfrak{a}} \in \delta^{\perp}$ and odd integer $c$. Then by direct computation, we get

$$
\bar{v}_{0}\left(\delta^{\prime}\right)-\bar{v}_{0}(\delta)=\bar{v}_{\delta}(\hat{\mathfrak{a}}) .
$$

This finishes the proof.

Theorem 3.4. Let $F$ be a hyperkähler manifold of $K 3^{[2]}$-type. Let $\bar{\delta} \in \mathrm{H}^{2}(F, \mathbb{Z} / 2 \mathbb{Z})$ be the canonical element as in Lemma 3.3(iii) and $\mathcal{K}^{2}(F)$ be the quotient of $\mathrm{H}^{2}(F, \mathbb{Z} / 2 \mathbb{Z})$ by $(\mathbb{Z} / 2 \mathbb{Z}) \bar{\delta}$. Then the following are true.

(i) There is a canonical short exact sequence

$$
0 \longrightarrow \mathcal{K}^{2}(F) \longrightarrow \mathcal{T}^{4}(F) \longrightarrow \mathbb{Z} / 10 \mathbb{Z} \longrightarrow 0
$$

Each $\delta \in \Omega(F)$ determines a splitting of the above sequence by the homomorphism $\mathbb{Z} / 10 \mathbb{Z} \rightarrow \mathcal{T}^{4}(F), 1 \mapsto \bar{v}_{0}(\delta)$.

(ii) There is a canonical exact sequence

$$
0 \longrightarrow \mathbb{Z} / 5 \mathbb{Z} \stackrel{\varphi}{\longrightarrow} \mathcal{T}^{4}(F) \stackrel{\psi}{\longrightarrow} \mathrm{H}^{2}(F, \mathbb{Z})^{\vee} \otimes \mathbb{Z} / 2 \mathbb{Z} \longrightarrow 0
$$

where $\varphi(1)=\bar{w}_{0}$ and $\psi(\theta)=\left\{\alpha \mapsto(\alpha \cdot \tilde{\theta} \cdot \delta)_{F} \bmod 2\right\}$, where $\tilde{\theta} \in \mathrm{H}^{4}(F, \mathbb{Z})$ is a lifting of $\theta \in \mathcal{T}^{4}(F)$ and $\delta \in \Omega(F)$ is some exceptional class. The composition $\psi \circ \bar{v}_{\delta}$ is the homomorphism induced by the Beauville-Bogomolov pairing. 
Proof. To prove (i), we note that by Lemma $3.3 \mathcal{K}^{2}(F)$ is identified with the image of $\bar{v}$. Since $\mathcal{K}^{2}(F)$ is of order $2^{22}$ and $\mathcal{T}^{4}(F)$ is of order $5 \cdot 2^{23}$, we know that the quotient group $\mathcal{T}^{4}(F) / \mathcal{K}^{2}(F)$ is of order 10 and thus isomorphic to $\mathbb{Z} / 10 \mathbb{Z}$. This gives the short exact sequence. By the explicit basis obtained in Theorem 2.17, we know that the images of $v_{0}$ and $\left\{v_{\delta}\left(\hat{\mathfrak{a}}_{i}\right)\right\}$ generate $\mathcal{T}^{4}(F)$. This implies that the element $\bar{v}_{0}$, which is of order 10 by Lemma 3.3 gives a splitting of the above short exact sequence.

To prove (ii), we first check that the definition of $\psi$ is independent of the choice of $\delta$. The composition $\psi \circ \bar{v}_{\delta}$ is induced by

$$
\alpha \mapsto\left\{\beta \mapsto\left(\frac{1}{2} \alpha(\alpha-\delta) \cdot \beta \cdot \delta\right)_{F} \bmod 2\right\} .
$$

Then we explicitly compute that

$$
\left(\frac{1}{2} \alpha(\alpha-\delta) \cdot \beta \cdot \delta\right)_{F} \equiv b(\alpha, \beta) \bmod 2 .
$$

This implies that $\psi \circ \bar{v}_{\delta}$ is the homomorphism induced by $b(-,-)$ whose image is

$$
\left\{\ell \in \operatorname{Hom}\left(\mathrm{H}^{2}(F, \mathbb{Z}), \mathbb{Z} / 2 \mathbb{Z}\right): \ell(\delta)=0, \forall \delta \in \Omega(F)\right\} .
$$

To show that $\psi$ is surjective, we only need to show that there exists an element $\theta \in \mathrm{H}^{4}(F, \mathbb{Z})$ such that $\theta \cdot \delta \cdot \delta \equiv 1 \bmod 2$. But we can simply take $\theta=v_{0}(\delta)$ since $v_{0}(\delta) \cdot \delta^{2}=-1$. The remaining part of (ii) follows from this.

\section{Minimal Hodge Classes and Picard RanK}

Let $F$ be a hyperkähler manifold of $K 3^{[2]}$-type. In this section we show that the minimal Hodge classes are of special form. Namely it is always contained in the subspace generated by divisors and the Beauville-Bogomolov form. In particular, under Assumption 1.4 we show that being of Jacobian type will force the Picard rank of $F$ to jump. As a consequence we see that a very general $F$ is not of Jacobian type. We also show that having a minimal Hodge class is a birational invariant.

4.1. Some canonical Hodge classes. Let $\left(F, \lambda_{0}\right)$ be a primitively polarized hyperkähler manifold of $K 3^{[2]}$-type. In this section, we study some canonical element in the group

$$
\operatorname{Hdg}^{4}(F)=\mathrm{H}^{4}(F, \mathbb{Z}) \cap \mathrm{H}^{2,2}(F)
$$

of Hodge classes in degree 4. First we note that there are two canonical classes in $\operatorname{Hdg}^{4}(F)$. The first one is $\left(\lambda_{0}\right)^{2}$ and the second one is constructed from the Beauville-Bogomolov form via linear algebra.

Let $\Lambda$ be a finitely geneated free abelian group with a nondegenerate integral symmetric bilinear form $b: \Lambda \times \Lambda \rightarrow \mathbb{Z}$. Let $\left\{\mathfrak{a}_{1}, \ldots, \mathfrak{a}_{r}\right\} \subset \Lambda$ be an integral basis of $\Lambda$. Let $A=\left(a_{i j}\right)_{r \times r}$ be the matrix representing the bilinear form $b$ with respect to the above basis. Namely $a_{i j}=b\left(\mathfrak{a}_{i}, \mathfrak{a}_{j}\right)$. Let $B=\left(b_{i j}\right)=A^{-1}$ and define

$$
b^{-1}=\sum_{i, j=1}^{r} b_{i j} \mathfrak{a}_{i} \mathfrak{a}_{j} \in \operatorname{Sym}^{2}(\Lambda) \otimes \mathbb{Q} .
$$

Note that $B$ is usually not integral unless the bilinear form $b$ is unimodular. Using standard linear algebra, one checks that $b^{-1}$ is independent of the choice of the basis $\left\{\mathfrak{a}_{i}\right\}$. Actually, we can choose $\left\{\mathfrak{a}_{i}\right\}$ to be a basis of $\Lambda_{\mathbb{Q}}=\Lambda \otimes \mathbb{Q}\left(\right.$ resp. $\left.\Lambda_{\mathbb{C}}=\Lambda \otimes \mathbb{C}\right)$ and extend $b$ linearly to $\Lambda_{\mathbb{Q}}$ (resp. $\Lambda_{\mathbb{C}}$ ), then the expression (8) gives the same element $b^{-1}$. 
Back to our specific situation, let $b(-,-)$ be the Beauville-Bogomolov bilinear form on $\mathrm{H}^{2}(F, \mathbb{Z})$. The above construction gives an element $b^{-1} \in \operatorname{Sym}^{2}\left(\mathrm{H}^{2}(F, \mathbb{Q})\right)$. By Lemma 2.1. this gives rise to an element $q \in \mathrm{H}^{4}(F, \mathbb{Q})$. We will frequently use the following explicit expression for $q$. Take an exceptional element $\delta \in \Omega(F)$ and let

$$
\left\{\hat{\mathfrak{a}}_{1}, \hat{\mathfrak{a}}_{2}, \ldots, \hat{\mathfrak{a}}_{22}\right\}
$$

be a basis of $\delta^{\perp}$. Let $A=\left(a_{i j}\right)$ be the matrix of $b$ restricted to $\delta^{\perp}$, i.e. $a_{i j}=b\left(\hat{\mathfrak{a}}_{i}, \hat{\mathfrak{a}}_{j}\right)$. Let $B=\left(b_{i j}\right)=A^{-1}$. Then we have

$$
q=\sum_{i, j} b_{i j} \hat{\mathfrak{a}}_{i} \hat{\mathfrak{a}}_{j}-\frac{1}{2} \delta^{2} \in \mathrm{H}^{4}(F, \mathbb{Q}) .
$$

Definition 4.1. Let $V_{\lambda_{0}} \subset \operatorname{Hdg}^{4}(F)$ be the subgroup defined by

$$
V_{\lambda_{0}}=\operatorname{Span}_{\mathbb{Q}}\left\{\lambda_{0}^{2}, q\right\} \cap \mathrm{H}^{4}(F, \mathbb{Z}) .
$$

Recall that $\lambda_{0}$ is even if $b\left(\lambda_{0}, \alpha\right)$ is even for all $\alpha \in \mathrm{H}^{2}(F, \mathbb{Z})$.

Lemma 4.2. Let $\lambda_{0} \in \mathrm{H}^{2}(F, \mathbb{Z})$ be primitive. The following statements are equivalent.

(i) The element $\lambda_{0}$ is even.

(ii) $\lambda_{0}^{2}-\delta^{2}$ is divisible by 2 for some $\delta \in \Omega(F)$.

(iii) $\lambda_{0}^{2}-\delta^{2}$ is divisible by 2 for all $\delta \in \Omega(F)$.

(iv) $\lambda_{0}^{2}-\delta^{2}$ is divisible by 8 for some $\delta \in \Omega(F)$.

(v) $\lambda_{0}^{2}-\delta^{2}$ is divisible by 8 for all $\delta \in \Omega(F)$.

(vi) $\bar{v}\left(\lambda_{0}\right)=0$ in $\mathcal{T}^{4}(F)$.

Proof. (i) $\Rightarrow$ (ii). Let $\delta \in \Omega(F)$ be some exceptional class. Fix a basis $\left\{\hat{\mathfrak{a}}_{1}, \ldots, \hat{\mathfrak{a}}_{22}\right\}$ of $\delta^{\perp}$. Then we can write $\lambda_{0}=\hat{\mathfrak{a}}^{\prime}+c^{\prime} \delta$ with $\hat{\mathfrak{a}}^{\prime} \in \delta^{\perp}$ and $c^{\prime} \in \mathbb{Z}$. The fact that $b\left(\lambda_{0}, \alpha\right) \in 2 \mathbb{Z}$ for all $\alpha \in \delta^{\perp}$ and the fact that $b(-,-)$ is unimodular on $\delta^{\perp}$ implies that $\hat{\mathfrak{a}}^{\prime}=2 \hat{\mathfrak{a}}$ for some $\hat{\mathfrak{a}} \in \delta^{\perp}$. Since $\lambda_{0}$ is primitive, we know that $c^{\prime}$ must be odd and hence we can write $\lambda_{0}=2 \hat{\mathfrak{a}}+(2 c+1) \delta$, for some $c \in \mathbb{Z}$. Then it is easy to see that $\lambda_{0}^{2}-\delta^{2}$ is divisible by 2 .

(ii) $\Rightarrow\left(\right.$ i). We still write $\lambda_{0}=\hat{\mathfrak{a}}^{\prime}+c^{\prime} \delta$. Then we get

$$
\lambda_{0}^{2}-\delta^{2}=\hat{\mathfrak{a}}^{\prime 2}+2 c^{\prime} \hat{\mathfrak{a}}^{\prime} \delta+\left(c^{\prime 2}-1\right) \delta^{2} .
$$

Using the explicit basis obtained in Theorem 2.13, we see that the above expression is divisible by 2 only if $2 \mid c^{\prime 2}-1$ and $\hat{\mathfrak{a}}^{\prime}=2 \hat{\mathfrak{a}}$ for some $\hat{\mathfrak{a}} \in \delta^{\perp}$. Hence we get $\lambda_{0}=2 \hat{\mathfrak{a}}+(2 c+1) \delta$ for some $c \in \mathbb{Z}$. This implies that $\lambda_{0}$ is even.

(ii) $\Rightarrow$ (iii) and (iv) $\Rightarrow(\mathrm{v})$. This is due to the fact that $\delta^{2}-\delta^{\prime 2}$ is divisible by 8 for all $\delta, \delta^{\prime} \in \Omega(F)$, see Lemma 2.14

(iii) $\Rightarrow$ (ii) and (v) $\Rightarrow$ (iv) are automatic.

(ii) $\Rightarrow$ (iv). As above, we can write $\lambda_{0}=2 \hat{\mathfrak{a}}+(2 c+1) \delta$. Hence we get

$$
\lambda_{0}^{2}-\delta^{2}=8 v_{\delta}(\hat{\mathfrak{a}})+8(c+1) \delta \hat{\mathfrak{a}}+4 c(c+1) \delta^{2},
$$

which is easily seen to be divisible by 8 .

(iv) $\Rightarrow\left(\right.$ vi). Again, we can write $\lambda_{0}=2 \hat{\mathfrak{a}}+(2 c+1) \delta$. Then we easily see that $\bar{v}\left(\lambda_{0}\right)=\bar{v}_{\delta}\left(\lambda_{0}\right)=0$ in $\mathcal{T}^{4}(F)$.

$($ vi $) \Rightarrow(\mathrm{i})$. Pick some $\delta \in \Omega(F)$. If we write $\lambda_{0}=\hat{\mathfrak{a}}^{\prime}+c^{\prime} \delta$ for some $\hat{\mathfrak{a}}^{\prime} \in \delta^{\perp}$ and $c^{\prime} \in \mathbb{Z}$, then $\bar{v}\left(\lambda_{0}\right)=\bar{v}\left(\hat{\mathfrak{a}}^{\prime}\right)=0$ implies that $\hat{\mathfrak{a}}^{\prime}$ is 0 in $\delta^{\perp} \otimes \mathbb{Z} / 2 \mathbb{Z}$, i.e. $\hat{\mathfrak{a}}^{\prime}=2 \hat{\mathfrak{a}}$ for some $\hat{\mathfrak{a}} \in \delta^{\perp}$. Since $\lambda_{0}$ is primitive, we get $c^{\prime}=2 c+1$ for some $c \in \mathbb{Z}$. This shows that $\lambda_{0}$ is even. 
Lemma 4.3. Let $\left(F, \lambda_{0}\right)$ be primitively polarized as above, then the following are true.

(i) The two elements $\left(\lambda_{0}\right)^{2}$ and $q$ are linearly independent in $\mathrm{H}^{2}(F, \mathbb{C})$.

(ii) For any $\alpha, \beta \in \mathrm{H}^{2}(F, \mathbb{Z})$, we have

$$
(q \cdot \alpha \cdot \beta)_{F}=25 b(\alpha, \beta) .
$$

(iii) The element $\frac{2}{5} q$ is integral and primitive, namely $\frac{2}{5} q \in \mathrm{H}^{4}(F, \mathbb{Z})$. Furthermore,

$$
\frac{2}{5} q+\delta^{2}=8 v_{0}(\delta), \quad \forall \delta \in \Omega(F) .
$$

(iv) If $\lambda_{0}$ is odd, then

$$
V_{\lambda_{0}}=\mathbb{Z}\left(\lambda_{0}\right)^{2} \oplus \mathbb{Z}\left(\frac{2}{5} q\right) .
$$

(v) If $\lambda_{0}$ is even, then $\left(\lambda_{0}\right)^{2}+\frac{2}{5} q$ is divisible by 8 and

$$
V_{\lambda_{0}}=\mathbb{Z}\left(\lambda_{0}\right)^{2} \oplus \mathbb{Z} \frac{1}{8}\left(\lambda_{0}^{2}+\frac{2}{5} q\right) .
$$

Proof. We fix an exceptional class $\delta \in \Omega(F)$ and let $\hat{\mathfrak{a}}_{i}, i=1, \ldots, 22$, be an integral basis of $\delta^{\perp}$. Let $A=\left(a_{i j}\right)_{1 \leq i, j \leq 22}$ be the matrix representing the restriction of $b$ to $\delta^{\perp}$. Let $B=\left(b_{i j}\right)=A^{-1}$. In particular, we have the explicit expression (9) for the class $q$.

We prove (ii) first by direct computation as follows. Assume that

$$
\alpha=a \delta+\sum x_{i} \hat{\mathfrak{a}}_{i}, \quad \beta=b \delta+\sum y_{i} \hat{\mathfrak{a}}_{i} .
$$

Then we have

$$
\begin{aligned}
(q \cdot \alpha \cdot \beta)_{F}= & \sum b_{i j}\left(\hat{\mathfrak{a}}_{i} \cdot \hat{\mathfrak{a}}_{j} \cdot \alpha \cdot \beta\right)_{F}-\frac{1}{2}\left(\delta^{2} \cdot \alpha \cdot \beta\right) \\
= & \sum b_{i j}\left(b\left(\hat{\mathfrak{a}}_{i}, \hat{\mathfrak{a}}_{j}\right) b(\alpha, \beta)+b\left(\hat{\mathfrak{a}}_{i}, \alpha\right) b\left(\hat{\mathfrak{a}}_{j}, \beta\right)+b\left(\hat{\mathfrak{a}}_{i}, \beta\right) b\left(\hat{\mathfrak{a}}_{j}, \alpha\right)\right) \\
& -\frac{1}{2} b(\delta, \delta) b(\alpha, \beta)-b(\alpha, \delta) b(\beta, \delta) \\
= & \left(\sum b_{i j} a_{i j}\right) b(\alpha, \beta)+2 \sum x_{k} a_{k i} b_{i j} a_{j l} y_{l}+b(\alpha, \beta)-4 a b \\
= & \operatorname{tr}(A B) b(\alpha, \beta)+b(\alpha, \beta)+2\left(\sum x_{k} a_{k l} y_{l}-2 a b\right) \\
= & 22 b(\alpha, \beta)+b(\alpha, \beta)+2 b(\alpha, \beta) \\
= & 25 b(\alpha, \beta) .
\end{aligned}
$$

To prove (i) we first note that for all $\alpha, \beta \in\left(\lambda_{0}\right)^{\perp}$, we have

$$
\left(\lambda_{0}^{2} \cdot \alpha \cdot \beta\right)_{F}=b\left(\lambda_{0}, \lambda_{0}\right) b(\alpha, \beta) .
$$

Hence if $\lambda_{0}^{2}$ and $q$ are proportional, then $\lambda_{0}^{2}=\frac{b_{0}}{25} q$ where $b_{0}=b\left(\lambda_{0}, \lambda_{0}\right)$. This implies

$$
3 b_{0}^{2}=\left(\lambda_{0}^{2} \cdot \lambda_{0}^{2}\right)=\frac{b_{0}}{25}\left(q \cdot \lambda_{0}^{2}\right)=\frac{b_{0}}{25} \cdot 25 b_{0}=b_{0}^{2} .
$$

This forces $b_{0}=0$ which is impossible since $\lambda_{0}$ is an ample class.

To prove (iii), we first compare the equation (7) and the equation (9) and get

$$
5 v_{0}(\delta)+q=\frac{5}{4} \sum_{i, j} b_{i j} \hat{\mathfrak{a}}_{i} \hat{\mathfrak{a}}_{j} .
$$


Note that $\frac{1}{2} \sum b_{i j} \hat{\mathfrak{a}}_{i} \hat{\mathfrak{a}}_{j}$ is integral. It follows that

$$
\frac{2}{5} q=\frac{1}{2} \sum_{i, j} b_{i j} \hat{\mathfrak{a}}_{i} \hat{\mathfrak{a}}_{j}-2 v_{0}(\delta)
$$

is integral and primitive. Equation (7) implies that $\frac{2}{5} q+\delta^{2}=8 v_{0}(\delta)$. This proves (iii).

The intersection matrix of $\left\{\lambda_{0}^{2}, \frac{2}{5} q\right\}$ is given by

$$
M=\left(\begin{array}{cc}
\lambda_{0}^{2} \cdot \lambda_{0}^{2} & \frac{2}{5} q \cdot \lambda_{0}^{2} \\
\frac{2}{5} q \cdot \lambda_{0}^{2} & \frac{2}{5} q \cdot \frac{2}{5} q
\end{array}\right)=\left(\begin{array}{cc}
3 b_{0}^{2} & 10 b_{0} \\
10 b_{0} & 92
\end{array}\right)
$$

Hence $\operatorname{det}(M)=176 b_{0}^{2}$. Now we assume that

$$
\frac{1}{p}\left(a \lambda_{0}+b\left(\frac{2}{5} q\right)\right) \in \mathrm{H}^{4}(F, \mathbb{Z})
$$

where $p$ is a prime and $a, b \in \mathbb{Z}$ with $\operatorname{gcd}(a, b)=1$. By Theorem 2.17, we have an integral basis

$$
\mathcal{B}=\left\{v_{0}(\delta), v_{\delta}\left(\hat{\mathfrak{a}}_{i}\right), \hat{\mathfrak{a}}_{i} \hat{\mathfrak{a}}_{j}, \delta \hat{\mathfrak{a}}_{i}\right\}
$$

for $\mathrm{H}^{4}(F, \mathbb{Z})$. We assume that $\lambda_{0}=k \hat{\mathfrak{a}}_{0}-c \delta$, for some $k, c \in \mathbb{Z}$ and primitive $\hat{\mathfrak{a}}_{0} \in \delta^{\perp}$. Let $\mu=b\left(\lambda_{0}, \delta\right)=2 c$ and $\mu_{i}=b\left(\lambda_{0}, \hat{\mathfrak{a}}_{i}\right)=k \tilde{\mu}_{i}$, where $\tilde{\mu}_{i}=b\left(\hat{\mathfrak{a}}_{0}, \hat{\mathfrak{a}}_{i}\right)$. The intersection of $\left\{\lambda_{0}^{2}, \frac{2}{5} q\right\}$ with the basis $\mathcal{B}$ is given by

$$
\left(\begin{array}{c}
\lambda_{0}^{2} \\
\frac{2}{5} q
\end{array}\right) \cdot \mathcal{B}=\left(\begin{array}{cccc}
k^{2} a_{0}-c^{2} & \mu_{i}\left(\mu_{i}-\mu\right)+\frac{a_{i i}}{2} b_{0} & 2 \mu_{i} \mu_{j}+b_{0} a_{i j} & 2 \mu \mu_{i} \\
9 & 5 a_{i i} & 10 a_{i j} & 0
\end{array}\right)
$$

where $a_{0}=b\left(\hat{\mathfrak{a}}_{0}, \hat{\mathfrak{a}}_{0}\right)$.

Claim: The fact that $p \mid a \lambda_{0}^{2}+b\left(\frac{2}{5} q\right)$ implies that $p \mid 4 k c$.

Proof of claim. Since both $\lambda_{0}^{2}$ and $\frac{2}{5} q$ are primitive, we know that $p \nmid a b$. The last column of (11) implies that $p \mid 4 k c \tilde{\mu}_{i}$, for all $i=1, \ldots, 22$. This forces $p \mid 2 k c$.

Case 1: $p \mid c$.

In this case we have

$$
b_{0}=b\left(\lambda_{0}, \lambda_{0}\right)=k^{2} b\left(\hat{\mathfrak{a}}_{0}, \hat{\mathfrak{a}}_{0}\right)-2 c^{2} \equiv k^{2} a_{0}, \quad \bmod p .
$$

The first column of (11) implies

$$
a k^{2} a_{0}+9 b \equiv 0 \quad \bmod p .
$$

By looking at the second column of (11), we get the following

$$
\begin{aligned}
0 & \equiv a \mu_{i}\left(\mu_{i}-\mu\right)+a \cdot \frac{a_{i i}}{2} b_{0}+b \cdot 5 a_{i i} \bmod p \\
& \equiv a k^{2} \tilde{\mu}_{i}^{2}+a \cdot \frac{a_{i i}}{2} k^{2} a_{0}+5 a_{i i} b \bmod p \\
& =a k^{2} \tilde{\mu}_{i}^{2}+\frac{a_{i i}}{2}\left(a k^{2} a_{0}+9 b\right)+\frac{a_{i i}}{2} b \\
& \equiv a k^{2} \tilde{\mu}_{i}^{2}+\frac{a_{i i}}{2} b \bmod p .
\end{aligned}
$$

This implies that $a_{i i}=c_{0} \tilde{\mu}_{i}^{2} \bmod p$, where $c_{0}=-\frac{2 a k^{2}}{b}$. Note that here we use the fact that $p \nmid b$. By looking at the third column of (11), we get

$$
\begin{aligned}
0 & \equiv a \cdot 2 k^{2} \tilde{\mu}_{i} \tilde{\mu}_{j}+a \cdot k^{2} a_{0} a_{i j}+10 b a_{i j} \quad \bmod p \\
& =2 a k^{2} \tilde{\mu}_{i} \tilde{\mu}_{j}+a_{i j}\left(a k^{2} a_{0}+9 b\right)+b a_{i j} \\
& \equiv 2 a k^{2} \tilde{\mu}_{i} \tilde{\mu}_{j}+b a_{i j} \quad \bmod p .
\end{aligned}
$$


It follows that $a_{i j}=c_{0} \tilde{\mu}_{i} \tilde{\mu}_{j} \bmod p$ for all $i<j$. Combine the above expressions for $a_{i j}$, we have

$$
\pm 1=\operatorname{det}(A) \equiv\left(c_{0}\right)^{22} \operatorname{det}\left(\tilde{\mu}_{i} \tilde{\mu}_{j}\right)=0 \quad \bmod p .
$$

This is a contradiction and hence case 1 never happens.

Case 2: $p \mid k$.

In this case, we first have $b_{0} \equiv-2 c^{2} \bmod p$. The first column of (11) gives

$$
-a c^{2}+9 b \equiv 0 \quad \bmod p .
$$

Then the third column of (11) gives

$$
\begin{aligned}
0 & \equiv 2 a \mu_{i} \mu_{j}+a b_{0} a_{i j}+10 b a_{i j} \quad \bmod p \\
& \equiv a\left(-2 c^{2}\right) a_{i j}+10 b a_{i j} \quad \bmod p \\
& =2 a_{i j}\left(-a c^{2}+9 b\right)-8 b a_{i j} \\
& \equiv-8 b a_{i j} \quad \bmod p
\end{aligned}
$$

Since $p \nmid b$, we have $p \mid 8 a_{i j}$ for all $i, j$. Since $\operatorname{det}(A)= \pm 1$, there exists $(i, j)$ such that $p \nmid a_{i j}$. It follows that $p \mid 8$ and hence $p=2$.

Case 3: $p=2$.

In this case, we have $2 \mid \lambda_{0}^{2}+\frac{2}{5} q$ and also $8 \mid \delta^{2}+\frac{2}{5} q$. By taking the difference, we see that $\lambda_{0}^{2}-\delta^{2}$ is divisible by 2 . By Lemma 4.2 we know that $\lambda_{0}$ is even. This implies that

$$
\lambda_{0}^{2}+\frac{2}{5} q=\left(\delta^{2}+\frac{2}{5} q\right)+\left(\lambda_{0}^{2}-\delta^{2}\right)
$$

is divisible by 8 . When $\lambda_{0}$ is even, we easily see that $b_{0} / 2=b\left(\lambda_{0}, \lambda_{0}\right) / 2$ is odd. Hence the power of the factor 2 in $\operatorname{det}(M)=176 b_{0}^{2}$ is 64 . It follows that

$$
\frac{1}{8}\left(\lambda_{0}^{2}+\frac{2}{5} q\right)
$$

is no longer divisible by 2 and hence is primitive. This proves (iv) and (v).

Corollary 4.4. If $\lambda_{0}$ satisfies the Assumption 1.4, then for all $\alpha \in V_{\lambda_{0}}$ and $\hat{\mathfrak{a}}, \hat{\mathfrak{a}}^{\prime} \in$ $\left(\lambda_{0}\right)^{\perp} \subset \mathrm{H}^{2}(F, \mathbb{Z})$, we have

$$
\left(\alpha \cdot \hat{\mathfrak{a}} \cdot \hat{\mathfrak{a}}^{\prime}\right)_{F}=e b\left(\hat{\mathfrak{a}}, \hat{\mathfrak{a}}^{\prime}\right),
$$

for some $e \in 2 \mathbb{Z}$. In particular, $V_{\lambda_{0}}$ contains no minimal Hodge classes.

Proof. We note that for all $\mathfrak{a}, \mathfrak{a}^{\prime} \in \mathrm{H}^{2}(F, \mathbb{Z})_{\mathrm{tr}}$, we have

$$
\frac{2}{5} q \cdot \mathfrak{a} \cdot \mathfrak{a}^{\prime}=10 b\left(\mathfrak{a}, \mathfrak{a}^{\prime}\right), \quad \lambda_{0}^{2} \cdot \mathfrak{a} \cdot \mathfrak{a}^{\prime}=b\left(\lambda_{0}, \lambda_{0}\right) b\left(\mathfrak{a}, \mathfrak{a}^{\prime}\right) .
$$

Then one concludes by evaluating $\mathfrak{a a}^{\prime}$ on the generators of $V_{\lambda_{0}}$.

\subsection{Minimal Hodge classes.}

Proposition 4.5. Let $F$ be a hyperkähler manifold of $K 3^{[2]}$-type. Then a minimal Hodge class $\theta$ is always in the $\mathbb{Q}$-vector space spanned by $\operatorname{Sym}^{2}(\operatorname{Pic}(F))$ and $q$.

Proof. Let $\left\{\mathfrak{a}_{1}, \ldots, \mathfrak{a}_{r}\right\}$ be a basis of $\operatorname{Hdg}^{2}(F)$ and $\left\{\mathfrak{t}_{1}, \ldots, \mathfrak{t}_{s}\right\}$ be an orthonormal basis of $\operatorname{Hdg}^{2}(F)^{\perp} \otimes \mathbb{C}$, i.e. $b\left(\mathfrak{t}_{\alpha}, \mathfrak{t}_{\beta}\right)=\delta_{\alpha \beta}, \forall \alpha, \beta \in\{1, \ldots, s\}$. Assume that $\theta \in \mathrm{H}^{4}(F, \mathbb{Z})$ is a minimal Hodge class. We can write $\theta$ explicitly as

$$
\theta=\sum_{i, j=1}^{r} x_{i j} \mathfrak{a}_{i} \mathfrak{a}_{j}+\sum_{i=1}^{r} \sum_{\alpha=1}^{s} y_{i \alpha} \mathfrak{a}_{i} \mathfrak{t}_{\alpha}+\sum_{\alpha, \beta=1}^{s} z_{\alpha \beta} \mathfrak{t}_{\alpha} \mathfrak{t}_{\beta},
$$


for some $x_{i j}, y_{i \alpha}, z_{\alpha \beta} \in \mathbb{C}$ with $x_{i j}=x_{j i}$ and $z_{\alpha \beta}=z_{\beta \alpha}$. Since $\theta$ is a minimal class, for all $\alpha, \beta \in\{1, \ldots, s\}$, we have

$$
\begin{aligned}
\delta_{\alpha \beta} & =\theta \cdot \mathfrak{t}_{\alpha} \cdot \mathfrak{t}_{\beta} \\
& =\sum_{i, j} x_{i j} b\left(\mathfrak{a}_{i}, \mathfrak{a}_{j}\right) \delta_{\alpha \beta}+\sum_{\alpha^{\prime}, \beta^{\prime}} z_{\alpha^{\prime} \beta^{\prime}}\left(\delta_{\alpha^{\prime} \beta^{\prime}} \delta_{\alpha \beta}+\delta_{\alpha^{\prime} \alpha} \delta_{\beta^{\prime} \beta}+\delta_{\alpha^{\prime} \beta} \delta_{\beta^{\prime} \alpha}\right) \\
& =\sum_{i j} x_{i j} b\left(\mathfrak{a}_{i}, \mathfrak{a}_{j}\right) \delta_{\alpha \beta}+\left(\sum_{\alpha^{\prime}} z_{\alpha^{\prime} \alpha^{\prime}}\right) \delta_{\alpha \beta}+2 z_{\alpha \beta} .
\end{aligned}
$$

When $\alpha \neq \beta$, we see that $z_{\alpha \beta}=0$; when $\beta=\alpha$, we get that $z_{\alpha \alpha}=c$ is a constant that is independent of $\alpha$. By definition, the element $q$ has the form

$$
q=\sum_{i, j} c_{i j} \mathfrak{a}_{i} \mathfrak{a}_{j}+\sum_{\alpha} \mathfrak{t}_{\alpha} \mathfrak{t}_{\alpha},
$$

which implies that $q_{\mathrm{tr}}=\sum_{\alpha} \mathfrak{t}_{\alpha} \mathfrak{t}_{\alpha}$ is of type $(2,2)$ in the Hodge decomposition. Now we take an integral basis $\left\{\tilde{\mathfrak{t}}_{1}, \ldots, \tilde{\mathfrak{t}}_{s}\right\}$ of $\mathrm{H}^{2}(F, \mathbb{Z})_{\mathrm{tr}}$. Hence we have a new explicit expression of $\theta$,

$$
\theta=\sum_{i, j=1}^{r} x_{i j} \mathfrak{a}_{i} \mathfrak{a}_{j}+\sum_{i=1}^{r} \sum_{\alpha=1}^{s} \tilde{y}_{i \alpha} \mathfrak{a}_{i} \tilde{\mathfrak{t}}_{\alpha}+c q_{\mathrm{tr}}
$$

where $\tilde{y}_{i \alpha}, c \in \mathbb{Q}$. Since $\theta$ is a Hodge class, we have

$$
\sum_{i=1}^{r} \sum_{\alpha=1}^{s} \tilde{y}_{i \alpha} \mathfrak{a}_{i} \tilde{\mathfrak{t}}_{\alpha}^{0,2}=0
$$

This implies that

$$
\sum_{\alpha=1}^{s} \tilde{y}_{i \alpha} \tilde{\mathfrak{t}}_{\alpha}^{0,2}=0
$$

since $\mathfrak{a}_{i}$ form a basis. Now it follows that $\sum_{\alpha=1}^{s} \tilde{y}_{i \alpha} \tilde{\mathfrak{t}}_{\alpha}$ is of type $(1,1)$ which forces it to be 0 . Hence we have $\theta=\sum_{i, j=1}^{r} x_{i j} \mathfrak{a}_{i} \mathfrak{a}_{j}+c q_{\text {tr }}$ which is in the $\mathbb{Q}$-span of $\operatorname{Sym}^{2}(\operatorname{Pic}(F))$ and $q$.

Corollary 4.6. Let $\left(F, \lambda_{0}\right)$ be a primitively polarized hyperkähler manifold of $K 3^{[2]}$ type with $\lambda_{0}$ satisfying Assumption 1.4. If $F$ is of Jacobian type, then the Picard number of $F$ is at least 2. In particular, a very general such $F$ is not of Jacobian type.

Proof. If $\operatorname{Pic}(F)=\mathbb{Z} \lambda_{0}$, then by the proposition a minimal Hodge class is always in $V_{\lambda_{0}}=\operatorname{Span}_{\mathbb{Q}}\left\{q, \lambda_{0}^{2}\right\} \cap \mathrm{H}^{2,2}(F)$. However, by Corollary 4.4, there is no such minimal Hodge class.

Proposition 4.7. Let $F_{1}$ and $F_{2}$ be hyperkähler manifolds of $K 3^{[2]}$-type. If $F_{1}$ is birational to $F_{2}$, then $F_{1}$ has a minimal Hodge class if and only if $F_{2}$ does.

Remark 4.8. This Proposition suggests that being of Jacobian type is likely to be a birational invariant.

Proof. Since $F_{1}$ and $F_{2}$ are birational to each other, we have have an isomorphism

$$
\mathrm{H}^{2}\left(F_{1}, \mathbb{Z}\right) \cong \mathrm{H}^{2}\left(F_{2}, \mathbb{Z}\right)
$$


which is compatible with the Beauville-Bogomolov bilinear forms; see [10]. Then Theorem 2.17 implies that

$$
\mathrm{H}^{4}\left(F_{1}, \mathbb{Z}\right) \cong \mathrm{H}^{4}\left(F_{2}, \mathbb{Z}\right)
$$

which is compatible with the intersections. Then the Proposition follows easily.

\section{Hodge Classes in Degree 4}

Let $F$ be a hyperkähler fourfold of $K 3^{[2]}$-type. In this section, we study Hodge classes in $\mathrm{H}^{4}(F)$ for a generic $F$ using a deformation argument. It is proved that all Hodge classes are generated by the polarization and the Beauville-Bogomolov form when $F$ is very general. This provides a second proof of the fact that a very general $F$ is not of Jacobian type. Since the Beauville-Bogomolov form is algebraic, our argument also gives a proof of the Hodge conjecture for very general $F$. In the special case when $F$ is the variety of lines on a very general cubic fourfold, we are able to prove the integral Hodge conjecture.

Theorem 5.1. Let $\left(F, \lambda_{0}\right)$ be a very general primitively polarized hyperkähler manifold of $K 3^{[2]}$-type. Then the group of integral Hodge classes in degree 4 can be expressed as

$$
\operatorname{Hdg}^{4}(F)=V_{\lambda_{0}} .
$$

In particular, if $\lambda_{0}$ is odd, then $\operatorname{Hdg}^{4}(F)$ is freely generated by $\lambda_{0}^{2}$ and $\frac{2}{5} q$; if $\lambda_{0}$ is even, then $\operatorname{Hdg}^{4}(F)$ is freely generated by $\lambda_{0}^{2}$ and $\frac{1}{8}\left(\lambda_{0}^{2}+\frac{2}{5} q\right)$.

Remark 5.2. Given Corollary 4.4, the above theorem gives another proof of the fact that if $\lambda_{0}$ satisfies Assumption 1.4 then a very general $F$ is not of Jacobian type.

Proof. Let $\Lambda=\mathrm{H}^{2}(F, \mathbb{Z})$. We will use $\Lambda_{\mathbb{C}}$ to denote $\Lambda \otimes \mathbb{C}$. Let $\mathcal{P}$ be the period map

$$
\mathcal{P}([F])=\mathbb{C} \omega_{F} \in \mathbb{P}\left(\Lambda_{\mathbb{C}}\right)
$$

where $\omega_{F} \in \mathrm{H}^{0}\left(F, \Omega_{F}^{2}\right)$ is a generator. By the Local Torelli Theorem (see [1]), we know that a deformation of $\left(F, \lambda_{0}\right)$ corresponds to a deformation of $\omega_{F}$ such that $\lambda_{0} \in \Lambda^{1,1}$. Consider

$$
Q_{\lambda_{0}}^{+}=\left\{\omega \in \mathbb{P}\left(\Lambda_{\mathbb{C}}\right): b(\omega, \omega)=0, b(\omega, \bar{\omega})>0, b\left(\lambda_{0}, \omega\right)=0\right\} .
$$

Assume that the period of $\left(F, \lambda_{0}\right)$ is $\omega$. Then we can easily compute

$$
T_{[\omega], Q_{\lambda_{0}}^{+}}=\left\{\varphi \in \operatorname{Hom}_{\mathbb{C}}\left(\mathbb{C} \omega, \Lambda_{\mathbb{C}} / \mathbb{C} \omega\right): b(\omega, \varphi(\omega))=0, b\left(\lambda_{0}, \varphi(\omega)\right)=0\right\} .
$$

By definition, $\varphi(\omega)$ is only a class in $\Lambda_{\mathbb{C}} / \mathbb{C} \omega$. Then we define $b(\omega, \varphi(\omega))$ to be $b\left(\omega, \omega^{\prime}\right)$ for any element $\omega^{\prime} \in \mathrm{H}^{2}(F, \mathbb{Z})$ representing the class $\varphi(\omega)$. Note that this is well defined since $b(\omega, \omega)=0$. We define $b\left(\lambda_{0}, \varphi(\omega)\right)$ in a similar way. We have the Hodge decomposition

$$
\Lambda_{\mathbb{C}}=\Lambda^{2,0} \oplus \Lambda^{1,1} \oplus \Lambda^{0,2} .
$$

Let $\left\{\alpha_{1}, \ldots, \alpha_{21}\right\}$ be a $\mathbb{C}$-basis of $\Lambda^{1,1}$. Then we can write

$$
\varphi(\omega)=\sum \mu_{i} \alpha_{i}+\mu_{0} \bar{\omega}, \quad \mu_{i}, \mu_{0} \in \mathbb{C} .
$$


The condition $b(\omega, \varphi(\omega))=0$ implies that $\mu_{0}=0$. The condition $b\left(\lambda_{0}, \varphi(\omega)\right)=0$, together with its equivalent form $b\left(\lambda_{0}, \overline{\varphi(\omega)}\right)=0$, implies that $\varphi(\omega) \in \lambda_{0}^{\perp} \cap \Lambda^{1,1}$. Hence we get the following identification

$$
T_{[\omega], Q_{\lambda_{0}}^{+}}=\lambda_{0}^{\perp} \cap \Lambda^{1,1}
$$

When $[\omega]$ moves in $Q_{\lambda_{0}}^{+}$along the direction $\varphi \in T_{[\omega], Q_{\lambda_{0}}^{+}}$, the space $\Lambda^{1,1}$ moves in the Grassmannian $G\left(21, \Lambda_{\mathbb{C}}\right)$ along the direction $\psi$, where

$$
\psi \in \operatorname{Hom}_{\mathbb{C}}\left(\Lambda^{1,1}, \mathbb{C} \omega \oplus \mathbb{C} \bar{\omega}\right)
$$

is the unique element satisfying

$$
\begin{aligned}
& b\left(\psi\left(\alpha_{i}\right), \omega\right)+b\left(\alpha_{i}, \varphi(\omega)\right)=0, \\
& b\left(\psi\left(\alpha_{i}\right), \bar{\omega}\right)+b\left(\alpha_{i}, \overline{\varphi(\omega)}\right)=0 .
\end{aligned}
$$

If we write $\psi\left(\alpha_{i}\right)=a_{i} \omega+b_{i} \bar{\omega}$, then the above conditions imply

$$
a_{i}=-b\left(\alpha_{i}, \overline{\varphi(\omega)}\right), \quad b_{i}=-b\left(\alpha_{i}, \varphi(\omega)\right) .
$$

When $[\omega]$ moves in $Q_{\lambda_{0}}^{+}$along the direction $\varphi$, the space $\mathrm{H}^{2,2}(F)$ moves in the Grassmannian $G\left(232, \operatorname{Sym}^{2}\left(\Lambda_{\mathbb{C}}\right)\right)$ along some direction $\tilde{\psi} \in \operatorname{Hom}_{\mathbb{C}}\left(\mathrm{H}^{2,2}, \operatorname{Sym}^{2}\left(\Lambda_{\mathbb{C}}\right) / \mathrm{H}^{2,2}\right)$. Under the natural identification

$$
\mathrm{H}^{2,2}=\operatorname{Sym}^{2}\left(H^{1,1}\right) \oplus \mathbb{C} \omega \wedge \bar{\omega},
$$

the homomorphism $\tilde{\psi}$ is given by

$$
\begin{aligned}
\tilde{\psi}\left(\alpha_{i} \alpha_{j}\right) & =\psi\left(\alpha_{i}\right) \wedge \alpha_{j}+\alpha_{i} \wedge \psi\left(\alpha_{j}\right), \\
\tilde{\psi}(\omega \wedge \bar{\omega}) & =\varphi(\omega) \wedge \bar{\omega}+\omega \wedge \overline{\varphi(\omega)} .
\end{aligned}
$$

We define the fixed part $\operatorname{Fix}\left(\mathrm{H}^{2,2}\right)$ of $\mathrm{H}^{2,2}$ to be the set of all $x \in \mathrm{H}^{2,2}$ such that for all $\tilde{\psi}$ associated to some $\varphi \in T_{[\omega], Q_{\lambda_{0}}^{+}}$, we have $\tilde{\psi}(x)=0$. Our next step is to determine $\operatorname{Fix}\left(\mathrm{H}^{2,2}\right)$ explicitly. Let $\varphi(\omega)=\sum \mu_{i} \alpha_{i}$ and $A=\left(a_{i j}\right)$ be the "intersection" matrix on $\mathrm{H}^{1,1}$, i.e. $a_{i j}=b\left(\alpha_{i}, \alpha_{j}\right)$. Let $T=\left(t_{i j}\right)$ be the matrix representing the complex conjugation on $\mathrm{H}^{1,1}$, i.e.

$$
\bar{\alpha}_{i}=\sum_{j=1}^{21} t_{i j} \alpha_{j} .
$$

Then we get

$$
\begin{aligned}
a_{i} & =-b\left(\alpha_{i}, \overline{\varphi(\omega)}\right) \\
& =-b\left(\alpha_{i}, \sum \bar{\mu}_{j} \bar{\alpha}_{j}\right) \\
& =-\sum \bar{\mu}_{j} t_{j k} b\left(\alpha_{i}, \alpha_{k}\right) \\
& =-\sum_{j, k} a_{i k} t_{j k} \bar{\mu}_{j}, \\
b_{i} & =-b\left(\alpha_{i}, \varphi(\omega)\right)=-\sum a_{i j} \mu_{j} .
\end{aligned}
$$

Let $\mathbf{a}=\left(a_{1}, \ldots, a_{21}\right)^{t}$ and $\mathbf{b}=\left(b_{1}, \ldots, b_{21}\right)^{t}$. Then the above equations can be written as

$$
\mathbf{a}=-A^{t} T \bar{\mu}, \quad \mathbf{b}=-A \mu
$$


where $\mu=\left(\mu_{1}, \ldots, \mu_{21}\right)^{t}$. Assume that

$$
x=\sum_{i, j} c_{i j} \alpha_{i} \alpha_{j}+c_{0} \omega \wedge \bar{\omega} \in \operatorname{Fix}\left(\mathrm{H}^{2,2}\right),
$$

where $C=\left(c_{i j}\right)$ is a symmetric matrix. Then we have

$$
\begin{aligned}
\tilde{\psi}(x) & =\sum_{i, j} c_{i j} \tilde{\psi}\left(\alpha_{i} \alpha_{j}\right)+c_{0} \tilde{\psi}(\omega \wedge \bar{\omega}) \\
& =\sum_{i j} c_{i j}\left(\alpha_{i} \wedge \psi\left(\alpha_{j}\right)+\psi\left(\alpha_{i}\right) \wedge \alpha_{j}\right)+c_{0}(\varphi(\omega) \wedge \bar{\omega}+\omega \wedge \overline{\varphi(\omega)}) \\
& =2\left({ }^{t} \alpha C \psi(\alpha)\right)+c_{0}(\varphi(\omega) \wedge \bar{\omega}+\omega \wedge \overline{\varphi(\omega)}) \\
& =2\left[{ }^{t} \alpha C(\mathbf{a} \omega+\mathbf{b} \bar{\omega})\right]+c_{0}\left({ }^{t} \alpha \mu \wedge \bar{\omega}+{ }^{t} \bar{\alpha} \bar{\mu} \wedge \omega\right) \\
& ={ }^{t} \alpha\left(-2 C A\left({ }^{t} T\right) \bar{\mu}+c_{0}\left({ }^{t} T\right) \bar{\mu}\right) \omega+{ }^{t} \alpha\left(-2 C A \mu+c_{0} \mu\right) \bar{\omega}
\end{aligned}
$$

where $\alpha=\left(\alpha_{1}, \ldots, \alpha_{21}\right)^{t}$ and $\psi(\alpha)=\left(\psi\left(\alpha_{1}\right), \ldots, \psi\left(\alpha_{21}\right)\right)^{t}$. Hence we get

$$
\begin{aligned}
x \in \operatorname{Fix}\left(\mathrm{H}^{2,2}\right) & \Leftrightarrow\left\{\begin{array}{l}
\left(c_{0}-2 C A\right) \mu=0 \\
\left(c_{0}-2 C A\right)^{t} T \bar{\mu}=0
\end{array} \quad \forall \varphi(\omega) \in \lambda_{0}^{\perp} \cap \Lambda^{1,1}\right. \\
& \Leftrightarrow\left(c_{0}-2 C A\right) \mu=0, \quad \forall \varphi(\omega) \in \lambda_{0}^{\perp} \cap \Lambda^{1,1} .
\end{aligned}
$$

We write $\lambda_{0}={ }^{t} \alpha \mathbf{s}$, where $\mathbf{s}=\left(s_{1}, \ldots, s_{21}\right)$, then

$$
\varphi(\omega) \in \lambda_{0}^{\perp} \cap \Lambda^{1,1} \Leftrightarrow{ }^{t} \mathbf{s} A \mu=0 .
$$

Hence we have the following equivalences

$$
\begin{aligned}
x \in \operatorname{Fix}\left(\mathrm{H}^{2,2}\right) & \Leftrightarrow\left(c_{0}-2 C A\right) \mu=0, \quad \forall \mu \text { with }{ }^{t} \mathbf{s} A \mu=0 \\
& \Leftrightarrow c_{0}-2 C A=\mathbf{t}^{t} \mathbf{s} A, \text { for some } \mathbf{t}=\left(t_{1}, \ldots, t_{21}\right)^{t} \\
& \Leftrightarrow C=\frac{1}{2} c_{0} A^{-1}-\frac{1}{2} \mathbf{t}^{t} \mathbf{s} .
\end{aligned}
$$

Since $C$ is symmetric, the vector $\mathbf{t}$ is a multiple of $\mathbf{s}$. Hence $x \in \operatorname{Fix}\left(\mathrm{H}^{2,2}\right)$ if and only if

$$
C=c_{1} B+c_{2} \mathbf{s}^{t} \mathbf{s}, \quad c_{0}=2 c_{1},
$$

for some $c_{1}, c_{2} \in \mathbb{C}$, where $B=A^{-1}$, i.e.

$$
\begin{aligned}
x & ={ }^{t} \alpha\left(c_{1} B+c_{2} \mathbf{s}^{t} \mathbf{s}\right) \alpha+2 c_{1} \omega \wedge \bar{\omega} \\
& =c_{1}\left({ }^{t} \alpha B \alpha+2 \omega \wedge \bar{\omega}\right)+c_{2} \lambda_{0}^{2} \\
& =c_{1} q+c_{2} \lambda_{0}^{2} .
\end{aligned}
$$

Hence we get $\operatorname{Fix}\left(\mathrm{H}^{2,2}\right)=\operatorname{Span}_{\mathbb{C}}\left\{q, \lambda_{0}^{2}\right\}$. This computation implies that on a very general deformation of $\left(F, \lambda_{0}\right)$, the classes that remain of $(2,2)$-type are generated by $q$ and $\lambda_{0}^{2}$. This implies that for a very general $\left(F, \lambda_{0}\right)$, we have

$$
\operatorname{Hdg}^{4}(F)=\operatorname{Span}_{\mathbb{Q}}\left\{q, \lambda_{0}^{2}\right\} \cap \mathrm{H}^{4}(F, \mathbb{Z}) .
$$

Together with Lemma 4.3, this proves the theorem.

Corollary 5.3. Let $\left(F, \lambda_{0}\right)$ be very general as in the theorem, then $\lambda_{0}$ is odd (resp. even) if and only if the image of $\operatorname{Hdg}^{4}(F)$ in $\mathcal{T}^{4}(F)$ is a cyclic group of order 5 (resp. 10). 
Proof. In any case, we always have $\lambda_{0}^{2} \mapsto 0 \in \mathcal{T}^{4}(F)$. Since $\frac{2}{5} q=8 v_{0}(\delta)-\delta^{2}$, we see that $\frac{2}{5} q \mapsto 8 \bar{v}_{0}$. If $\lambda_{0}$ is odd, then the image of $\operatorname{Hdg}^{4}(F)$ in $\mathcal{T}^{4}(F)$ is generated by $8 \bar{v}_{0}$, which is an element of order 5 . To conclude the second case, we only need to note the fact that for any even $\lambda_{0}$, the image of $\frac{1}{8}\left(\lambda_{0}^{2}-\delta^{2}\right)$ in $\mathcal{T}^{4}(F)$ is an element of order 2 .

Corollary 5.4. Let $\left(F, \lambda_{0}\right)$ be a very general polarized hyperkähler manifold of $K 3^{[2]}$-type.

(i) The Hodge conjecture holds true for $F$.

(ii) Let $\mathcal{Z}^{4}(F)=\operatorname{Hdg}^{4}(F) / \operatorname{Alg}^{4}(F)$. If $\lambda_{0}$ is odd, then there is a surjection $\mathbb{Z} / 3 \mathbb{Z} \rightarrow$ $\mathcal{Z}^{4}(F)$; if $\lambda_{0}$ is even, then there is a surjection $\mathbb{Z} / 24 \mathbb{Z} \rightarrow \mathcal{Z}^{4}(F)$.

Proof. To prove the Hodge conjecture for $F$, we only need to do this in degree 4. By the computation carried out in the proof of the theorem, we only need to show that $q$ is algebraic. We will need the following explicit formula of the Chern classes of $F$ in the special case $F=S^{[2]}$,

$$
c_{2}(F)=24 v_{0}-3 \delta^{2} .
$$

See [22, Lemma 9.3]. This implies that $\frac{2}{5} q=\frac{1}{3} c_{2}(F)$. When $\lambda_{0}$ is odd, then $\mathcal{Z}^{4}(F)$ is generated by the image of $\frac{1}{3} c_{2}(F)$. When $\lambda_{0}$ is even, then $\mathcal{Z}^{4}(F)$ is generated by the image of $\frac{1}{24} c_{2}(F)+\frac{1}{8} \lambda_{0}^{2}$. This proves the corollary.

We consider a special family of polarized hyperkähler manifolds. Let $X \subset \mathbb{P}_{\mathbb{C}}^{5}$ be a smooth cubic fourfold. Let $F=F(X)$ be the variety of lines on $X$. It is known that $F$ is a hyperkähler manifold of $K 3^{[2]}$-type; see [2, Proposition 2]. We have a natural inclusion $F \subset G(2,6)$. Let $\mathscr{E}$ be the restriction of the rank 2 quotient bundle on the Grassmannian of lines in $\mathbb{P}^{5}$. Set $g_{1}=c_{1}(\mathscr{E})$ and $g_{2}=c_{2}(\mathscr{E})$. We take $\lambda_{0}=g_{1}$ to be the natural polarization on $F$. Recall from Hassett [8] that a cubic fourfold $X$ is special if $\operatorname{Hdg}^{4}(X)$ has rank at least two. Beauville-Donagi 2, Proposition 4] proved that the Abel-Jacobi homomorphism $\Phi: \mathrm{H}^{4}(X, \mathbb{Z}) \rightarrow$ $\mathrm{H}^{2}(F, \mathbb{Z})$ is an isomorphism of Hodge structures. Hence $X$ is special if and only if the Picard rank of $F$ is at least two.

Proof of Proposition 1.7. We only need to verify Assumption 1.4 for one cubic fourfold $X$. We take $X$ to be general Pfaffian cubic fourfold. Then $F=S^{[2]}$ for some $K 3$ surface $S$ of degree 14 . Let $\delta \in \operatorname{Pic}(F)$ be the boundary divisor and $\mathfrak{b} \in \operatorname{Pic}(S)$ be the polarization with $(\mathfrak{b} \cdot \mathfrak{b})_{S}=14$. Then under the natural orthogonal decomposition

$$
\mathrm{H}^{2}(F, \mathbb{Z})=\mathrm{H}^{2}(S, \mathbb{Z}) \oplus \mathbb{Z} \delta, \quad b(\delta, \delta)=-2,
$$

the polarization $\lambda_{0}$ can be written as $\lambda_{0}=2 \mathfrak{b}-5 \delta$; see the proof of [2, Proposition 6]. Then Assumption 1.4 is readily verified. Then by Corollary 4.6, we have $\operatorname{rk} \operatorname{Pic}(F) \geq$ 2. Hence $X$ is special.

Theorem 5.5. Let $X$ be a very general cubic fourfold. Then the following are true.

(i) The variety of lines $F=F(X)$ is not of Jacobian type.

(ii) The integral Hodge classes $\operatorname{Hdg}^{4}(F)$ is freely generated by $g_{2}$ and $\frac{1}{3}\left(g_{1}^{2}-g_{2}\right)$.

(iii) The integral Hodge conjecture holds true for $F$ in degree 4.

Proof. (i) Follows from the above proposition since a very general $X$ is not special. To prove (ii), we need to write $q$ explicitly in terms of $g_{1}$ and $g_{2}$. Let 
$\Phi: \mathrm{H}^{4}(X, \mathbb{Z}) \rightarrow \mathrm{H}^{2}(F, \mathbb{Z})$ be the Abel-Jacobi isomorphism. Then the restriction of $\Phi$ to the transcendental classes gives an isomorphism

$$
\Phi_{0}: \mathrm{H}^{4}(X, \mathbb{Z})_{\mathrm{tr}} \rightarrow \mathrm{H}^{2}(F, \mathbb{Z})_{\mathrm{tr}}
$$

which satisfies $b(\Phi(\alpha), \Phi(\beta))=-\alpha \cdot \beta$ for all $\alpha, \beta \in \mathrm{H}^{4}(X, \mathbb{Z})_{\mathrm{tr}}$; see 2, Proposition $6]$. One relation that we need is

$$
g_{2} \cdot \mathfrak{a} \cdot \mathfrak{a}^{\prime}=0, \quad \forall \mathfrak{a}, \mathfrak{a}^{\prime} \in \mathrm{H}^{2}(F, \mathbb{Z})_{\mathrm{tr}} .
$$

This can be seen from the geometry. Let $Y \subset X$ be a general hyperplane section, which is a smooth cubic threefold. Then $g_{2}$ is represented by the surface of lines on $Y$. For any transcendental classes $\mathfrak{a}$ and $\mathfrak{a}^{\prime}$, we can find the corresponding transcendental classes $\alpha, \alpha^{\prime} \in \mathrm{H}^{4}(X, \mathbb{Z})_{\text {tr }}$ such that $\mathfrak{a}=\Phi(\alpha)$ and $\mathfrak{a}^{\prime}=\Phi\left(\alpha^{\prime}\right)$. When $\alpha$ is transcendental, we always have $\operatorname{deg}\left(\left.\alpha\right|_{Y}\right)=0$. By the formula of the number of secant lines of a pair of curves on a cubic hypersurface obtained in [20, Lemma 3.10], we have

$$
g_{2} \cdot \mathfrak{a} \cdot \mathfrak{a}^{\prime}=5 \operatorname{deg}\left(\left.\alpha\right|_{Y}\right) \operatorname{deg}\left(\left.\alpha^{\prime}\right|_{Y}\right)=0 .
$$

Similarly, we have $g_{2} \cdot g_{1} \cdot \mathfrak{a}=0$ and $g_{2} g_{1}^{2}=45$. The self-intersection of $g_{1}$ is given by

$$
g_{1}^{4}=3 b\left(g_{1}, g_{1}\right)^{2}=108 .
$$

Based on these identities, we get

$$
\left(\frac{1}{6} g_{1}^{2}-\frac{4}{15} g_{2}\right) \cdot \mathfrak{a} \cdot \mathfrak{b}=b(\mathfrak{a}, \mathfrak{b}), \quad \forall \mathfrak{a}, \mathfrak{b} \in \mathrm{H}^{2}(F, \mathbb{Z}) .
$$

This implies that $\frac{1}{6} g_{1}^{2}-\frac{4}{15} g_{2}=\frac{1}{25} q$. Hence we have

$$
\frac{1}{8}\left(\frac{2}{5} q+g_{1}^{2}\right)=\frac{1}{3}\left(g_{1}^{2}-g_{2}\right)
$$

Then (ii) follows from Theorem 5.1 and the fact that $g_{1}$ is even.

Note that $\frac{1}{3}\left(g_{1}^{2}-g_{2}\right)$ is represented by the surface of all lines meeting a given line, see [24, §0]. This proves (iii).

\section{Rational CUBic Fourfolds}

In this section, we give some evidence of our Conjecture 1.6. We first state some well-known formulas for blow-ups. We then show that rationality of a fourfold implies the existence of a Hodge theoretically special family of rational curves parametrized by a surface. Using the techniques and constructions of [20], we were able to relate this to the variety of lines and prove Theorem 1.10 .

6.1. Blow up formulas. Let $Y$ be a smooth projective variety of dimension 4 and $Z \subset Y$ a smooth closed subvariety. Let $\tilde{Y}=\operatorname{Bl}_{Z}(Y)$ be the blow up of $Y$ along $Z$ and $E \subset \tilde{Y}$ the exceptional divisor. Then we have the following commutative diagram

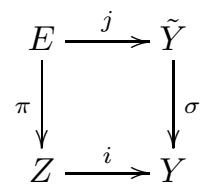


Proposition 6.1. Let $Y, Z$ and $\tilde{Y}$ be as above. We use $\hat{\oplus}$ to denote orthogonal decomposition. Then the following are true.

(i) If $Z$ is a point, then

$$
\mathrm{H}^{4}(\tilde{Y}, \mathbb{Z}) \cong \mathrm{H}^{4}(Y, \mathbb{Z}) \hat{\oplus} \mathbb{Z}\left[\mathbb{P}^{2}\right],
$$

where the extra class $\left[\mathbb{P}^{2}\right]$ is the class of a linear $\mathbb{P}^{2}$ in the exceptional divisor $E \cong \mathbb{P}^{3}$. Furthermore we have $\left[\mathbb{P}^{2}\right]^{2}=-1$.

(ii) If $Z$ is a curve, then

$$
\mathrm{H}^{4}(\tilde{Y}, \mathbb{Z})=\mathrm{H}^{4}(Y, \mathbb{Z}) \hat{\oplus}\left(\mathbb{Z} j_{*} \xi \oplus \mathbb{Z} j_{*} f\right),
$$

where $\xi$ is the class of the relative $\mathcal{O}(1)$-bundle of $\pi: E=\mathbb{P}\left(\mathscr{N}_{Z / Y}\right) \rightarrow Z$ and $f$ is the class of a fiber of $\pi$. The intersection matrix of $j_{*} \xi$ and $j_{*} f$ is

$$
\left(\begin{array}{cc}
d & -1 \\
-1 & 0
\end{array}\right)
$$

where $d=\operatorname{deg}\left(\mathscr{N}_{Z / Y}\right)$.

(iii) If $Z$ is a surface, then we have a canonical identification

$$
\mathrm{H}^{4}(\tilde{Y}, \mathbb{Z})=\mathrm{H}^{4}(Y, \mathbb{Z}) \hat{\oplus} \mathrm{H}^{2}(Z, \mathbb{Z})(-1),
$$

where $\mathrm{H}^{2}(Z, \mathbb{Z}) \rightarrow \mathrm{H}^{4}(\tilde{Y}, \mathbb{Z})$ is given by $\mathfrak{a} \mapsto-j_{*} \pi^{*} \mathfrak{a}$ and the intersection form is given by

$$
j_{*} \pi^{*} \mathfrak{a} \cdot j_{*} \pi^{*} \mathfrak{a}^{\prime}=-\left(\mathfrak{a} \cdot \mathfrak{a}^{\prime}\right)_{Z} .
$$

The projection $\mathrm{H}^{4}(\tilde{Y}, \mathbb{Z}) \rightarrow \mathrm{H}^{2}(Z, \mathbb{Z})$ is given by $\alpha \mapsto \pi_{*} j^{*} \alpha$.

Sketch of Proof. These formulas are classical. For example (see $\S 2.1$ of [18]), one can use the Mayer-Vietoris sequence to get the following short exact sequence

$$
0 \longrightarrow \mathrm{H}^{4}(Y, \mathbb{Z}) \stackrel{\left(i^{*}, \sigma^{*}\right)}{\longrightarrow} \mathrm{H}^{4}(Z, \mathbb{Z}) \oplus \mathrm{H}^{4}(\tilde{Y}, \mathbb{Z}) \stackrel{\pi^{*}-j^{*}}{\longrightarrow} \mathrm{H}^{4}(E, \mathbb{Z}) \longrightarrow 0 .
$$

Since $E$ is a projective bundle over $Z$, we can write $\mathrm{H}^{4}(E)$ in terms of the cohomology of $Z$. Then the formulas (without the bilinear form) in the proposition follow from the above sequence. To get the bilinear form, it remains to carry out a routine computation of intersection numbers using the Chern class of the relative $\mathcal{O}(1)$-bundle of $E \rightarrow Z$. Please also see the proof of Lemma 2.4.

Corollary 6.2. If $Z$ is a point or a curve, then

$$
\mathrm{H}^{4}(\tilde{Y}, \mathbb{Z})_{\operatorname{tr}}=\mathrm{H}^{4}(Y, \mathbb{Z})_{\mathrm{tr}} .
$$

If $Z$ is a surface, then

$$
\mathrm{H}^{4}(\tilde{Y}, \mathbb{Z})_{\operatorname{tr}}=\mathrm{H}^{4}(Y, \mathbb{Z})_{\operatorname{tr}} \hat{\oplus} \mathrm{H}^{2}(Z, \mathbb{Z})_{\operatorname{tr}}(-1) .
$$

6.2. Rational fourfolds. Let $Y$ be a smooth projective fourfold that is rationally connected. Let $S=\amalg S_{i}$ be a smooth (not necessarily irreducible) surface. Let $K(S)=\oplus K\left(S_{i}\right)$ be the product of the function fields of its components. Any nontrivial morphism

$$
\varphi_{K(S)}: \mathbb{P}_{K(S)}^{1}=\coprod \mathbb{P}_{K\left(S_{i}\right)}^{1} \rightarrow Y
$$

induces an Abel-Jacobi map

$$
\alpha=\pi_{*} \varphi^{*}: \mathrm{H}^{4}(Y, \mathbb{Z})_{\mathrm{tr}} \rightarrow \mathrm{H}^{2}(S, \mathbb{Z})_{\mathrm{tr}} .
$$


This can be defined as follows. First, the morphism $\varphi$ can be defined over a dense open subset $U \subset S$. Namely, we have the following diagram

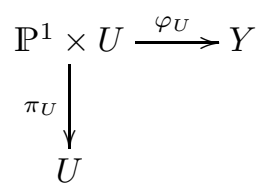

This picture can be completed into a proper family of rational curves on $Y$ as follows

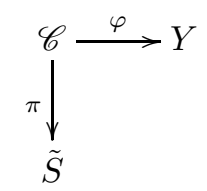

This allows us to define the usual Abel-Jacobi map

$$
\pi_{*} \varphi^{*}: \mathrm{H}^{4}(Y, \mathbb{Z})_{\mathrm{tr}} \rightarrow \mathrm{H}^{2}(\tilde{S}, \mathbb{Z})_{\mathrm{tr}} .
$$

It is well-known that the transcendental lattice of a surface is a birational invariant. Since $S$ is birational to $\tilde{S}$, we have the canonical isomorphism

$$
\mathrm{H}^{2}(\tilde{S}, \mathbb{Z})_{\mathrm{tr}} \cong \mathrm{H}^{2}(S, \mathbb{Z})_{\mathrm{tr}} .
$$

This gives the homomorphism

$$
\alpha: \mathrm{H}^{4}(Y, \mathbb{Z})_{\operatorname{tr}} \rightarrow \mathrm{H}^{2}(S, \mathbb{Z})_{\operatorname{tr}} .
$$

One checks that this definition of $\alpha$ is independent of the choice of the spreading $\varphi_{U}$ and the completion $\tilde{S}$. Note that by construction, the morphism $\pi: \mathscr{C} \rightarrow \tilde{S}$ has a rational section.

Definition 6.3. We say that $S$ receives the cohomology of $Y$ with index $e$ via a rational curve $\varphi_{K(S)}: \mathbb{P}_{K(S)}^{1} \rightarrow Y$ as above if the associated Abel-Jacobi map satisfies

$$
(\alpha(x) \cdot \alpha(y))_{S}=-e(x \cdot y)_{Y}, \quad \forall x, y \in \mathrm{H}^{4}(Y, \mathbb{Z})_{\operatorname{tr}} .
$$

When a polarization $H$ of $Y$ is fixed, the degree of the rational curve $\varphi_{K(S)}$ is defined as the degree of $\varphi\left(\mathbb{P}_{s}^{1}\right)$ for a general closed point $s \in S(\mathbb{C})$. We will call $\varphi_{K(S)}$ a line if its degree is 1 .

Let $Y$ be a smooth projective fourfold. Assume that $Y$ is rational. Then there is a birational map $f: \mathbb{P}^{4} \rightarrow Y$. We resolve the indeterminacy of $f$ by successive blow-ups along smooth centers and get the following picture

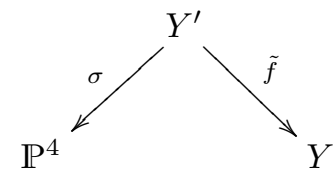

where $\sigma$ is the seccessive blow-up. Let $S_{1}, \ldots, S_{r}$ be the smooth surfaces that appear as the center of the blow-up at some step. Let $T_{1}, \ldots, T_{r} \subset \mathbb{P}^{4}$ be the images of the $S_{i}$ 's.

Definition 6.4. We say that successive blow-up $\sigma$ is simple if the $T_{i}$ 's are all surfaces distinct from each other. 
We assume that $\sigma$ is simple. This implies that $S_{i} \rightarrow T_{i}$ is birational and $\sigma^{-1}(t) \cong$ $\mathbb{P}^{1}$ for general $t \in T_{i}$. Let

$$
S=\coprod_{i=1}^{r} S_{i}, \quad T=\coprod_{i=1}^{r} T_{i} .
$$

Fix a general point $x \in Y$ with its pre-image in $\mathbb{P}^{4}$ being $x^{\prime}$. For a general point (meaning from a dense open subset) $t \in T$, we use $L_{t} \subset \mathbb{P}^{4}$ to denote the line passing through $x^{\prime}$ and $t$. Let $L_{t}^{\prime} \subset Y^{\prime}$ be the strict transform of $L_{t}$ and $C_{t} \subset Y$ be its image in $Y$. Note that $x \in C_{t}$ for all such general $t$. As $t$ varies in a dense open subset of $S$, this gives a rational curve

$$
\varphi_{K(S)}: \mathbb{P}_{K(S)}^{1} \rightarrow Y
$$

that passes through $x \in X$. For a general point $t \in T$, we also define $E_{t}^{\prime}=\sigma^{-1}(t) \subset$ $Y^{\prime}$ and $E_{t} \subset Y$ be its image in $Y$. Since $S \rightarrow T$ is birational, we see that $E_{t}$, as $t$ runs through general points of $T$, defines a rational curve

$$
\tilde{\varphi}_{K(S)}: \mathbb{P}_{K(S)}^{1} \rightarrow Y \text {. }
$$

Lemma 6.5. Let notations and assumptions be as above, then $S$ receives the cohomology of $Y$ via $\varphi_{K(S)}$ (resp. $\left.\tilde{\varphi}_{K(S)}\right)$ with index -1 (resp. 1).

Proof. By (iii) of Proposition 6.1 and Corollary 6.2, we see that

$$
\tilde{\pi}_{*} \tilde{\varphi}^{*}: \mathrm{H}^{4}(Y, \mathbb{Z})_{\operatorname{tr}} \rightarrow \mathrm{H}^{2}(S, \mathbb{Z})_{\operatorname{tr}}=\mathrm{H}^{4}\left(Y^{\prime}, \mathbb{Z}\right)_{\operatorname{tr}}
$$

is equal to $\tilde{f}^{*}$. This proves the case of $\tilde{\varphi}_{K(S)}$. Let $\left\{\tilde{L}_{b}: b \in B=\mathbb{P}^{3}\right\}$ be all the lines on $\mathbb{P}^{4}$ passing through $x^{\prime}$. Then for general $b \in B$, the line $\tilde{L}_{b}$ gives rise to a rational curve $C_{b}^{\prime} \subset Y$. When $\tilde{L}_{b}$ specializes to a line $L_{t}$ for some general point $t \in T$, the rational curve $C_{b}^{\prime}$ specializes to the nodal curve $C_{t} \cup E_{t}$. This gives us the following picture

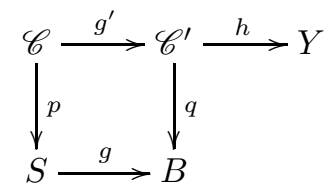

Here $\mathscr{C}$ is the total space of $\left\{E_{t} \cup C_{t}\right\}_{t \in S}$ and $\mathscr{C}^{\prime}$ is the total space of $\left\{\tilde{L}_{b}\right\}_{b \in B}$. Then by construction, we have

$$
\pi_{*} \varphi^{*}+\tilde{\pi}_{*} \tilde{\varphi}^{*}=p_{*}\left(g^{\prime}\right)^{*} h^{*}=g^{*} q_{*} h^{*}
$$

as homomorphisms $\mathrm{H}^{4}(Y, \mathbb{Z})_{\mathrm{tr}} \rightarrow \mathrm{H}^{2}(S, \mathbb{Z})_{\mathrm{tr}}$. Since $\mathrm{H}^{2}(B, \mathbb{Z})_{\mathrm{tr}}=0$, we get $g^{*}=0$ and hence

$$
\pi_{*} \varphi^{*}=-\tilde{\pi}_{*} \tilde{\varphi}^{*}
$$

This proves the case of $\varphi_{K(S)}$.

6.3. Rational cubic fourfolds. Let $X$ be a smooth cubic fourfold and $F=F(X)$ its variety of lines.

Proposition 6.6. The hyperkähler manifold $F$ is of Jacobian type if and only if there exists a surface $S$ that receives the cohomology of $X$ with index 1 via lines. 
Proof. By [2, Proposition 4], the universal line defines the Abel-Jacobi isomorphism

$$
\Phi: \mathrm{H}^{4}(X, \mathbb{Z}) \rightarrow \mathrm{H}^{2}(F, \mathbb{Z}) .
$$

If there is a line $\varphi_{K(S)}: \mathbb{P}_{K(S)}^{1} \rightarrow X$, then this gives a rational map $f: S \rightarrow F$. By replacing $S$ by a birational model, we may assume that $f$ is a morphism. Then we have

$$
f^{*} \circ \Phi=\pi_{*} \varphi^{*}: \mathrm{H}^{4}(X, \mathbb{Z})_{\mathrm{tr}} \rightarrow \mathrm{H}^{2}(S, \mathbb{Z})_{\mathrm{tr}}
$$

As was shown in [2, Proposition 6],

$$
b(\Phi(x), \Phi(y))=-(x \cdot y), \quad x, y \in \mathrm{H}^{4}(X, \mathbb{Z})_{\mathrm{tr}} .
$$

We see that $S$ represents a minimal class if and only if it receives cohomology of $X$ with index 1 via $\varphi_{K(S)}$.

Remark 6.7. We have two well-known examples of rational cubic fourfolds. The first example is the Pfaffian cubic fourfolds and the second example is the cubic fourfolds containing a plane where a certain Brauer element vanishes. We will show that Conjecture 1.6 holds in each example.

Let $X$ be a general Pfaffian cubic fourfolds. It was proved by Beauville-Donagi 2. that $X$ is rational and the corresponding variety $F$ of lines is isomorphic to $S^{[2]}$ for some $K 3$ surface $S$. Thus our Conjecture 1.6 holds for such $X$.

Let $X$ be a general cubic fourfold containing a plane $\Pi \cong \mathbb{P}^{2} \subset X$. Let $\tilde{X}$ be the blow-up of $X$ along $\Pi$. The projection from the plane $\Pi$ determines a morphism $\pi: \tilde{X} \rightarrow \Pi^{\prime}=\mathbb{P}^{2}$. A general fiber of $\pi$ is a smooth quadric surface and hence isomorphic to $\mathbb{P}^{1} \times \mathbb{P}^{1}$. There is a smooth curve $\Delta \subset \Pi^{\prime}$ of degree 6 such that for all $t \in \Delta$ the fiber $\pi^{-1} t$ is isomorphic to the cone over a conic. Note that a general fiber of $\pi$ has two rulings and by singling out one of them, we get a double cover $r: S \rightarrow \Pi^{\prime}$ which ramifies along $\Delta$. Hence $S$ is a $K 3$ surface of degree 2. Consider the actual lines in a given ruling, we get a divisor $D \subset F$ which has a natural morphism $s: D \rightarrow S$ whose closed fibers are all isomorphic to $\mathbb{P}^{1}$. Associated to this situation, we have an element $\alpha \in \operatorname{Br}(S)$ that corresponds to the fibration $D \rightarrow S$. Now we assume that $\alpha=0$ and consequently $s$ admits a rational section $\lambda: S \rightarrow D$. In this case, $X$ is rational since the vanishing of $\alpha$ implies that $\pi$ has a rational section and hence $\tilde{X}$ is rational; see [9]. By blowing up $S$, we get a morphism $\tilde{\lambda}: \tilde{S} \rightarrow D$. Together with the natural inclusion $D \subset F$, we get a surface $\phi: \tilde{S} \rightarrow F$. We next show that $\tilde{S}$ represents a minimal class on $F$ and thus Conjecture 1.6 is verified in this case. In $\mathrm{H}^{4}(X, \mathbb{Z})$, we have two natural classes, namely $\Pi$ and $h^{2}$. Let $L$ be the orthogonal complement of $\left\langle h^{2}, \Pi\right\rangle$ in $\mathrm{H}^{4}(X, \mathbb{Z})$. Voisin [24, Proposition 2] proved that $\left.\left.\Phi(L)\right|_{D} \subset s^{*} \mathrm{H}^{(} S, \mathbb{Z}\right)^{0}$ where $\mathrm{H}^{2}(S, \mathbb{Z})^{0}$ is the orthogonal complement of $r^{*} c_{1}\left(\mathcal{O}_{\Pi^{\prime}}(1)\right)$ in $\mathrm{H}^{2}(S, \mathbb{Z})$. Furthermore, Voisin also proved that if $x, y \in L$ and if $x^{\prime}, y^{\prime} \in \mathrm{H}^{2}(S, \mathbb{Z})^{0}$ such that $\left.\Phi(x)\right|_{D}=$ $s^{*} x^{\prime},\left.\Phi(y)\right|_{D}=s^{*} y^{\prime}$, then $(x \cdot y)_{X}=-\left(x^{\prime} \cdot y^{\prime}\right)_{S}$. By [2, Proposition 6], we have $b(\Phi(x), \Phi(y))=-(x \cdot y)_{X}$. It follows that

$$
\left(\phi^{*} u \cdot \phi^{*} v\right)_{\tilde{S}}=\left(\tilde{\lambda}^{*} s^{*} x^{\prime} \cdot \tilde{\lambda}^{*} s^{*} y^{\prime}\right)_{\tilde{S}}=\left(x^{\prime} \cdot y^{\prime}\right)_{S}=b(u, v),
$$

for all $u=\Phi(x)$ and $v=\Phi(y)$ in $\Phi(L)$, where $x, y \in L$. Note that $\mathrm{H}^{2}(F, \mathbb{Z})_{\operatorname{tr}} \subset$ $\Phi(L)$. Hence the above equality implies that $\phi_{*}[\tilde{S}]$ is minimal class.

Proposition 6.8. Let $C \cong \mathbb{P}^{1} \subset X$ be a general rational curve of degree $e$ on $X$ and $S_{C}$ the surface of lines meeting $C$. Then the lines defined by $S_{C}$ gives a morphism $\varphi_{K(S)}: \mathbb{P}_{K(S)}^{1} \rightarrow X$ and $S_{C}$ receives the cohomology of $X$ with index $2 e$. 
Proof. This follows from the Prym-Tjurin construction obtained in [21].

Given the above result, we would like to ask the following

Question 6.9. For a very general cubic fourfold $X$, is there a surface $S$ that receives the cohomology of $X$ via a rational curve with an odd index?

We expect that the answer to this question is negative and such a negative answer should imply nonrationality of $X$. To see how this question is related to Conjecture 1.6. we recall some constructions and results of [20. Let $f: C=\mathbb{P}^{1} \rightarrow X$ be a smooth rational curve of degree $e$ on $X$. A secant line of $C$ is a line on $X$ that meets $C$ in 2 points. We say that $C$ is well-positioned if there are only finitely many distinct secant lines of $C$. Assume that $C$ is well-positioned. A general pair of points $(x, y) \in \operatorname{Sym}^{2}(C)=\mathbb{P}^{2}$ determines a line $L_{x, y} \subset \mathbb{P}^{5}$. The line $L_{x, y}$ intersects $X$ in a third point $z$ unless $L_{x, y}$ is a secant line of $C$. As $(x, y)$ varies, we get a rational map $\phi_{0}: \operatorname{Sym}^{2}(C) \rightarrow X,(x, y) \mapsto z$. Let $\Sigma$ be the blow up of $\operatorname{Sym}^{2}(C)$ at the points corresponding to the secant lines, then $\phi_{0}$ extends to a morphism $\phi: \Sigma \rightarrow X$. This rational surface $\Sigma$ is called the residue surface of $C$. The condition $z=x$ or $y$ defines a curve $\tilde{C} \subset \Sigma$. The restriction of $\phi$ to $\tilde{C}$ gives a morphism $\tilde{C} \rightarrow C$ which has degree $e-2$. There are several natural divisors on $\Sigma$. Let $x \in C$ be a general closed point, we define $D_{x} \subset \Sigma$ be the locus $\left\{\left(x, x^{\prime}\right): x^{\prime} \in C\right\} \subset \operatorname{Sym}^{2}(C)$. Let $\mathfrak{a} \subset C$ be a hyperplane section and we write $\mathfrak{a}=\sum_{i=1}^{e} x_{i}$. We define

$$
D_{\mathfrak{a}}=\sum_{i=1}^{e} D_{x_{i}} .
$$

Let $\xi=\phi^{*} h$ be the class of a hyperplane. The locus $(x, x)$ for $x \in C$ defines $\Delta \subset \Sigma$. The class of $\Delta$ is divisible by 2 in the Picard group of $\Sigma$. Let $\Delta_{0}=-\frac{1}{2} \Delta$. Then the following holds true in $\operatorname{Pic}(\Sigma)$,

$$
\tilde{C}-D_{\mathfrak{a}}-\Delta_{0}-\xi+\sum_{i=1}^{N} E_{i}=0,
$$

where $E_{i}, i=1, \ldots, N$, are all the exceptional divisors of the blow up $\Sigma \rightarrow \operatorname{Sym}^{2}(C)$ and $\mathfrak{a}=\left.h\right|_{C}$. This is proved in Proposition 3.6 of [20. Viewed as algebraic cycles on $X$ modulo rational equivalence, we have

$$
\phi_{*} \tilde{C}=(e-2) C, \quad \phi_{*} D_{\mathfrak{a}}=-e C+a_{1} h^{3}, \quad \phi_{*} \Delta_{0}=C+a_{2} h^{3}, \quad \phi_{*} \xi=a_{3} h^{3},
$$

for some $a_{1}, a_{2}, a_{3} \in \mathbb{Z}$; see the proof of [20, Theorem 4.2].

If $C$ varies in a family, then the above constructions can be carried out in families. Furthermore, the construction does not require the base field to be algebraically closed. Now assume that $S$ is a surface and

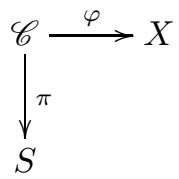

be a family of rational curves of degree $e$ on $X$. Assume that for a general point $s \in S$, the curve $\mathscr{C}_{s}$ is well-positioned. By carrying out the above construction in this family, we see that there is a dense open subset $U \subset S$ and $\tilde{\Sigma} \rightarrow U, \tilde{\mathscr{C}} \subset \tilde{\Sigma}$, $\Xi \subset \tilde{\Sigma}, \tilde{D} \subset \tilde{\Sigma}, \tilde{\Delta}_{0} \subset \tilde{\Sigma}$ and $\tilde{E} \subset \tilde{\Sigma}$ such that for any point $s \in U$, the fiber $(\tilde{\Sigma})_{s}$ is the residue surface of $\mathscr{C}_{s}$, and $\tilde{\mathscr{C}}, \Xi, \tilde{D}, \tilde{\Delta}_{0}$ and $\tilde{E}$ correspond to $\tilde{C}, \xi, D_{\mathfrak{a}}, \Delta_{0}$ and 
$\sum E_{i}$ respectively. We view $\tilde{\mathscr{C}}, \Xi, \tilde{D}, \tilde{\Delta}_{0}$ and $\tilde{E}$ as elements in $\mathrm{CH}_{3}(\tilde{\Sigma})$. Then we define

$$
\tilde{\Gamma}=\tilde{\mathscr{C}}-\tilde{D}-\tilde{\Delta}_{0}-\Xi+\tilde{E} \in \mathrm{CH}_{3}(\tilde{\Sigma}) .
$$

Note that there is a natural morphism $\tilde{\phi}: \tilde{\Sigma} \rightarrow U \times X$. We define $\Gamma \in \mathrm{CH}_{3}(S \times X)$ to be the closure of $\tilde{\phi}_{*} \tilde{\Gamma}$. By abuse of notation, we will simply write $\Gamma=\tilde{\phi}_{*} \tilde{\Gamma}$. Let $\eta_{S}$ be the generic point of $S$. Note that $\left.\Gamma\right|_{\eta_{S} \times X}$ defines an element in $\mathrm{CH}_{1}\left(\eta_{S} \times X\right)$. By doing the above construction of residue surface over the point $\eta_{S}$, then the relation (12) says

$$
\left.\Gamma\right|_{\eta_{S} \times X}=0
$$

Note that we have

$$
\mathrm{CH}_{1}\left(\eta_{S} \times X\right)=\mathrm{CH}^{3}\left(\eta_{S} \times X\right)=\underset{\lim }{\longrightarrow} \mathrm{CH}^{3}(U \times X)
$$

where $U$ runs through open dense subsets of $S$. Hence we see that $\left.\Gamma\right|_{U \times X}=0$ for some open dense $U \subset S$. Let $B=S-U$ and $\tilde{B}$ be a desingularization of $B$. By shrinking $U$, we may assume that $B$ is a curve. Then we see that

$$
\Gamma \in \operatorname{Im}\left(\mathrm{CH}_{3}(\tilde{B} \times X) \rightarrow \mathrm{CH}_{3}(S \times X)\right) .
$$

It follows that the homomorphism $[\Gamma]^{*}: \mathrm{H}^{4}(X, \mathbb{Z})_{\operatorname{tr}} \rightarrow \mathrm{H}^{2}(S, \mathbb{Z})_{\text {tr }}$ factors through $\mathrm{H}^{0}(\tilde{B}, \mathbb{Z})$. This implies that $[\Gamma]^{*}=0$. Note that $\tilde{\phi}_{*} \tilde{\mathscr{C}}=(e-2) \mathscr{C} \in \mathrm{CH}_{3}(S \times X)$, we get

$$
\left[\tilde{\phi}_{*} \tilde{\mathscr{C}}\right]^{*}=(e-2)[\mathscr{C}]^{*} .
$$

Consider $\Gamma_{1}=\tilde{\phi}_{*}\left(\tilde{D}+e \tilde{C}-\frac{a_{1}}{a_{3}} \Xi\right) \in \mathrm{CH}_{3}(S \times X)_{\mathbb{Q}}$ and run the same argument as for $\Gamma$, we get

$$
\left[\tilde{\phi}_{*} \tilde{D}\right]^{*}=-e[\mathscr{C}]^{*}+\frac{a_{1}}{a_{3}}\left[\tilde{\phi}_{*} \Xi\right]^{*} .
$$

Similarly, we have

$$
\left[\tilde{\phi}_{*} \tilde{\Delta}_{0}\right]^{*}=[\mathscr{C}]^{*}+\frac{a_{2}}{a_{3}}\left[\tilde{\phi}_{*} \Xi\right]^{*} .
$$

Note that $\Xi$ is simply the pull back of a hyperplane $H \subset X$ via the natural morphism $\tilde{\Sigma} \rightarrow X$. It follows that $\left[\tilde{\phi}_{*} \Xi\right]^{*}: \mathrm{H}^{4}(X, \mathbb{Z})_{\operatorname{tr}} \rightarrow \mathrm{H}^{2}(S, \mathbb{Z})_{\text {tr }}$ factors through $\mathrm{H}^{4}(H, \mathbb{Z})$. Since $\mathrm{H}^{4}(H, \mathbb{Z}) \cong \mathbb{Z}$ only has algebraic classes, we conclude that

$$
\left[\tilde{\phi}_{*} \Xi\right]^{*}=0
$$

Put all these identities together, we get

$$
[\tilde{E}]^{*}=(3-2 e)[\mathscr{C}]^{*}, \quad \text { on } \mathrm{H}^{4}(X, \mathbb{Z})_{\mathrm{tr}} .
$$

The generic fiber of $\tilde{E} \rightarrow S$ consists of finitely many lines, namely the secant lines of the corresponding curve $\mathscr{C}_{s}$. By selecting one of the secant lines, we get a factorization $\tilde{E} \rightarrow \Gamma_{S} \rightarrow S$ and a morphism $\Gamma_{S} \rightarrow F$. The surface $\Gamma_{S}$ gives rise to a correspondence from $S$ to $F$. Then the equation (13) can be rewritten as

$$
\left[\Gamma_{S}\right]^{*} \circ \alpha=(3-2 e)[\mathscr{C}]^{*},
$$

where $\alpha: \mathrm{H}^{4}(X, \mathbb{Z})_{\operatorname{tr}} \rightarrow \mathrm{H}^{2}(F, \mathbb{Z})_{\operatorname{tr}}$ is the Abel-Jacobi isomorphism. Now we can give a partial relation between Question 6.9 and Conjecture 1.6.

Proposition 6.10. Let $X$ be a cubic fourfold and $F$ its variety of lines. Assume that there exist a surface $S$ receiving the cohomology of $X$ with odd index $e_{0}$ via a well-positioned rational curve. Then $F$ is potentially of Jacobian type. 
Proof. Let $\mathscr{C} \rightarrow S$ be the rational curve defined by $S$. By assumption, we have

$$
[\mathscr{C}]^{*} x \cdot[\mathscr{C}]^{*} y=-e_{0}(x \cdot y), \quad x, y \in \mathrm{H}^{4}(X, \mathbb{Z})_{\operatorname{tr}} .
$$

Let $\Gamma_{S}$ be the surface parameterizing the secant lines as constructed above. Then the equation (14) gives

$$
\left[\Gamma_{S}\right]^{*} u \cdot\left[\Gamma_{S}\right]^{*} v=e_{0}(2 e-3) b(u, v), \quad u, v \in \mathrm{H}^{2}(F, \mathbb{Z})_{\mathrm{tr}} .
$$

Note that $e_{0}(2 e-3)$ is again odd. Take $a=\frac{e_{0}(2 e-3)-1}{2} \in \mathbb{Z}$. Let $l \subset X$ be a general line and $f: S_{l} \rightarrow F$ the surface of lines meeting $l$. It is known that

$$
f^{*} u \cdot f^{*} v=2 b(u, v), \quad u, v \in \mathrm{H}^{2}(F, \mathbb{Z})_{\mathrm{tr}} .
$$

Then we can take $S_{1}=S \coprod S_{l}$ and $\Gamma_{1}=\Gamma_{S}-a \Gamma_{f}$, where $\Gamma_{f}$ is the graph of $f$. Then we have

$$
\left[\Gamma_{1}\right]^{*} u \cdot\left[\Gamma_{1}\right]^{*} v=b(u, v), \quad u, v \in \mathrm{H}^{2}(F, \mathbb{Z})_{\mathrm{tr}} .
$$

This means that $F$ is potentially of Jacobian type.

Proof of Theorem 1.10. This is essentially a combination of Lemma 6.5 and Proposition 6.10. The only thing that we need to note is that in the proof of Lemma 6.5. we can choose the point $x^{\prime}$ general enough such that $C_{t}$ is well-positioned for general $t$.

\section{REFERENCES}

[1] A. Beauville, Variétés kählériennes dont la première classe de Chern est nulle, J. of Diff. Geometry, 18(1983), 755-782.

[2] A. Beauville and R. Donagi, La variété des droites d'une hypersurface cubique de dimension 4, C.R. Acad. Sc. Paris, 301(1985), 703-706.

[3] F. Bogomolov, On the cohomology ring of a simple hyperkähler manifold (on the results of Verbitsky), Geom. Funct. Anal., 6 (1996), pp.612-618.

[4] S. Boissière, M. Nieper-Wisskirchen and A. Sarti, Smith theory and irreducible holomorphic symplectic manifolds, Journal of Topology (to appear).

[5] H. Clemens and P. Griffiths, The intermediate Jacobian of a cubic threefold, Ann. Math., 95(1972), 281-356.

[6] W. Fulton, Intersection theory, second edition, Springer 1998.

[7] A. Grothendieck, Sur quelques points d'algébre homologique, Tôhoku Math. J. (2) 9, 1957, $119-221$.

[8] B. Hassett, Special cubic fourfolds, Compositio Math. 120(2000), no. 1, 1-23.

[9] B. Hassett, Some rational cubic fourfolds, J. Algebraic Geom. 8 (1999), no. 1, 103-114.

[10] D. Huybrechts, Compact hyperkähler manifolds: Basic results, Invent. Math. 135 (1999), 63-113.

[11] E. Izadi, A Prym construction for the cohomology of a cubic hypersurface, Proc. of L.M.S., 79(1999), 535-568.

[12] A. Kuznetsov, Derived categories of cubic fourfolds, in "Cohomological and geometric approaches to rationality problems", 219243, Progr. Math., 282, Birkhuser Boston, Boston, 2010.

[13] V.S. Kulikov, A remark on the nonrationality of a generic cubic fourfold, (Russian) Mat. Zametki 83 (2008), no. 1, 61-68; translation in Math. Notes 83 (2008), no. 1-2, 57-64.

[14] W. Li and Z. Qin, Integral cohomology of Hilbert schemes of points on surfaces, Comm. in Anal. and Geom. 16, no.5, 2008, 969-988.

[15] E. Markman, Integral generators for the cohomology ring of moduli spaces of sheaves over Poisson surfaces, Adv. Math. 208 (2007), no. 2, 622-646.

[16] E. Markman and S. Mehrotra, Hilbert schemes of K3 surfaces are dense in moduli, arXiv: 1201.0031.

[17] T. Matsusaka, On a characterization of a Jacobian variety, Mem. College Sci. Univ. Kyoto Ser. A Math. Volume 32, Number 1 (1959), 1-19.

[18] C. Peters, Tata lectures on motivic aspects of Hodge theory. 
[19] Z. Qin and W. Wang, Integral operators and integral cohomology classes of Hilbert schemes, Math. Ann. 331, 2005, 669-692.

[20] M. Shen, On relations among 1-cycles on cubic hypersurfaces, J. Alg. Geom. (to appear).

[21] M. Shen, Prym-Tjurin constructions on cubic hypersurfaces, preprint. arXiv:1111.6327

[22] M. Shen and C. Vial, The Fourier transform for certain hyperkähler fourfolds, preprint. arXiv:1309.5965

[23] M. Verbitsky, Cohomology of compact hyperkähler manifolds and its applications, Goem. Funct. Anal., 6 (1996), pp. 601-611.

[24] C. Voisin, Théorème de Torelli pour les cubiques de $\mathbb{P}^{5}$, Invent. Math. 86(1986), 577-601.

[25] E. C. Zeeman, Dihomology. III. A generalization of the Poincaré duality for manifolds, Proc. London Math. Soc. (3) 13, 1963, p.155-183.

DPMms, University of Cambridge, Wilberforce Road, Cambridge CB3 0WB, UK

E-mail address: M.Shen@dpmms.cam.ac.uk 